\title{
Long ties accelerate noisy threshold-based contagions
}

\author{
Dean Eckles $^{\mathrm{a}, \mathrm{b}, 1}$, Elchanan Mossel ${ }^{\mathrm{b}, \mathrm{c}, 1}$, M. Amin Rahimian ${ }^{\mathrm{b}, 1}$, and Subhabrata Sen ${ }^{\mathrm{c}, 1}$ \\ a Sloan School of Management; ${ }^{\mathrm{b}}$ Institute for Data, Systems, and Society; ${ }^{\mathrm{c}}$ Department of Mathematics, Massachusetts \\ Institute of Technology, Cambridge, MA 02139
}

Working paper. May 24, 2019.

\begin{abstract}
Network structure can affect when and how widely new ideas, products, and behaviors are adopted. In widely-used models of biological contagion, interventions that randomly rewire edges (generally making them "longer") accelerate spread. However, there are other models relevant to social contagion, such as those motivated by myopic best-response in games with strategic complements, in which an individual's behavior is described by a threshold number of adopting neighbors above which adoption occurs (i.e., complex contagions). Recent work has argued that highly clustered, rather than random, networks facilitate spread of these complex contagions. Here we show that minor modifications to this model, which make it more realistic, reverse this result: we allow very rare below-threshold adoption, i.e., rarely adoption occurs when there is only one adopting neighbor. To model the trade-off between long and short edges we consider networks that are the union of cycle-power- $k$ graphs and random graphs on $n$ nodes. Allowing adoptions below threshold to occur with order $1 / \sqrt{n}$ probability along some "short" cycle edges is enough to ensure that random rewiring accelerates spread. Simulations illustrate the robustness of these results to other commonly-posited models for noisy bestresponse behavior. Hypothetical interventions that randomly rewire existing edges or add random edges (versus adding addition of "short", triad-closing edges) in hundreds of empirical social networks reduce time to spread. This revised conclusion suggests that those wanting to increase spread should induce formation of long ties, rather than triad-closing ties. More generally, this highlights the importance of noise in game-theoretic analyses of behavior.
\end{abstract}

Keywords: social networks | game dynamics | social contagion

Authors are listed in alphabetical order. D.E., E.M., M.A.R., and S.S. designed research, performed research, contributed new analytic tools, analyzed data, and wrote the paper.

D.E. previously had a significant financial interest in Facebook while conducting this research. E.M., M.A.R. and S.S. declare no conflict of interest.

${ }^{1}$ To whom correspondence should be addressed: \{eckles,elmos,rahimian,ssen90\}@mit.edu 
How does network structure affect the spread of ideas, products, and behaviors? Social interactions among individuals facilitate a diverse range of contagions, and understanding the role of contact structure is central to all social and behavioral sciences. Decision-makers often rely on their knowledge of contagion in planning interventions that seed a behavior (1-5), prevent or reverse infection of nodes (6-8), or that attempt to modify network structure (9-12). Unfortunately, existing analyses of the two most widely used families of models (simple and complex contagions) have led to opposing conclusions (weakness or strength of long ties) about how network structure — in particular, clustering — affects spread of behavior.

Social contagions that are expected to be driven by incidental transfer of information are often modeled by analogy to biological contagion of infectious disease. In such simple contagion models, a node has an independent (and typically identical) probability of being infected by each infected neighbor (13); see Figure 1A. It is well known that such contagions spread more slowly in highly clustered networks than in more random networks $(14,15)$. Related considerations lead to the "strength of weak ties" hypothesis by which "weak" (or more properly "long") ties play critical roles in access to information (16), such as in labor markets (cf. 17). On the other hand, adoptions which are costly, or occur because of normative social pressure or coordination, are often modeled as myopic best-responses in repeated graphical games of strategic complementarities (such as coordination games), whereby nodes' utilities from adopting depend on the number of adopting neighbors (18-21). Threshold activation functions are the archetypal example of such complex contagion models $(22,23)$. In their canonical form, a single parameter $\theta$ divides non-adoption from adoption such that adoption is non-existent when the number of adopting neighbors is below the threshold $\theta$ (Figure 1B); call this deterministic $\theta$-complex contagion. Recent analyses of this deterministic model (23), or limits of noisy best replies as the noise level goes to zero (24) ( $q, \sigma \rightarrow 0$ in Figure 1B), have concluded that the spread of complex contagions is facilitated by more clustered networks, such that there is a "weakness of long ties" (23).*

Is such a deterministic model realistic? Empirical studies of social contagion, including those that ostensibly provide evidence for complex contagion, find substantial probability of adoption with a single adopting neighbor (1, 25-27). Empirical adoption rates with $k$ and $k-1$ neighboring adopters often have similar magnitudes: in illustrative recent studies, adoption rates with an additional adopter (beyond the first) increase by less than a factor of five (SI Appendix, Figure S1). Rather than positing determinism, analyses of discrete choice problems typically hypothesize that individuals are random utility maximizers leading to positive choice probabilities over the entire support. Specific noise distributions then rationalize choice probabilities specified by, e.g., probit or logit functions (Figure 1B).

Here we show that allowing a small probability of below-threshold adoption (denoted by $q$ in Figure 1B), even only via some short ties, reverses existing stylized facts about how network structure affects the spread of complex contagions. This harmonizes theoretical guidance about how network structure affects the spread of both simple and complex contagions.

\footnotetext{
*Indeed, an extreme case is straightforward: consider the spread of deterministic, 2-complex contagion on the 2-regular circular lattice structure shown in the middle Figure 2. Infecting any two adjacent nodes on the circle will cause the entire graph to be infected after four time steps. However, rewiring any of the edges that is not connecting the initial infected nodes ("seeds") and replacing it with a long tie across the circle prevents the global spread of the deterministic, 2-complex contagion.
} 


\section{Comparison to Related Work}

Our results fall in the general category of work that addresses how network structure affects social contagions $(21,24,28)$. This question has been studied by researchers in many fields, including applied probability $(29,30)$, computer science (31-34), physics (35), economics (36), organization science (37) and sociology (23).

Janson et al. (29) study the threshold spreading process (known in applied probability as bootstrap percolation) using a mean-field approximation. They use a random graph model that is the union of lattice with random (long) edges. The lattice structure provides for local clustering, while the random (long) edges decrease the network diameter. Both these features (high clustering and a small diameter) are observed in real network data and are the basis for the Watts-Strogatz small-world random graph model (14), where the edges of a cycle-power- $k$ graph (denoted by $\mathcal{C}_{k}$, see Figure 2 ) are rewired and replaced by random long ties. The Watts-Strogatz model is suitable for studying the effect of network structure and interventions that modify local clustering. It is often useful to consider the closely related and more analytically amenable Newman-Watts model (38) in which random edges are added on top of $\mathcal{C}_{k}$. Our first result establishes the rate of spread over $\mathcal{C}_{2}$ union random graphs and tightens the existing upper and lower bounds for rate of spread over the Newman-Watts random graphs (31). Our second and third results allow us to provide a more refined picture for cases with partial rewiring of the cycle structure. The spread of threshold processes have been also analyzed in a variety of other random graph models, including random regular graphs, power-law and configuration models (32-34, 39-41). In other related work (20,42), researchers analyze how the network structure affects the equilibria of coordination games, where agents best respond to the fraction of their adopting neighbors. The concept of cohesiveness proposed by Morris (20) is useful for characterizing the final adopter set. Accordingly, highly cohesive (and clustered) groups with many internal edges are difficult to penetrate, impeding the spread of fractional threshold models.

We are interested in realistic models of complex contagion which allow for a non-zero probability of adoption below threshold. Focusing on the canonical case of 2-complex contagion ${ }^{\dagger}$, we denote the probability of (simple) adoptions with a single neighboring adopter by $q_{n}$, where $n$ is the network size. Our characterization is in terms of time to global spread. We show that having $q^{\ddagger} q_{n}=\omega(1 / \sqrt{n})$ is enough to make contagion spread faster over the rewired graph; thus changing the landscape of results leading to weakness of long ties for complex contagions. Our theoretical analyses and simulation results indicate that long ties accelerate realistic complex (i.e., threshold-based) contagions.

\footnotetext{
${ }^{\dagger}$ In a $d$-regular graph, 2-complex contagion corresponds to a fractional threshold model $(20,22)$ with fixed (relative) thresholds set to $2 / d$. Using an absolute threshold allows us to isolate the effect of structural interventions irrespective of the initial seed sets. For example, introduction of a new edge has a monotone increasing influence on the spreading rate in an absolute threshold model; whereas, if thresholds are specified with respect to the ratio of adopters in each neighborhood, then depending on the size and location of adopters one can place the new edges carefully to impede the spread.

${ }^{\ddagger}$ Given three functions $f(\cdot), g(\cdot)$, and $h(\cdot)$ we use the asymptotic notations $f(n)=O(g(n))$ and $f(n)=o(h(n))$ to signify the relations $\limsup _{n \rightarrow \infty}|f(n) / g(n)|<\infty$ and $\lim _{n \rightarrow \infty}|f(n) / h(n)|=0$, respectively; in the latter case we also write $h(n)=\omega(f(n))$. We use $f(n)=\Omega(g(n))$ to signify $\liminf _{n \rightarrow \infty} f(n) / g(n)>0$. We use $f(n)=\Theta(g(n))$ to mean that $f(n)=O(g(n))$ and $f(n)=\Omega(g(n))$. We sometimes describe the asymptotic orders up to a logarithmic factor and use $O^{*}(f(n))$ to mean $O\left(f(n) \log ^{\alpha}(n)\right)$ for some fixed $\alpha$.
} 


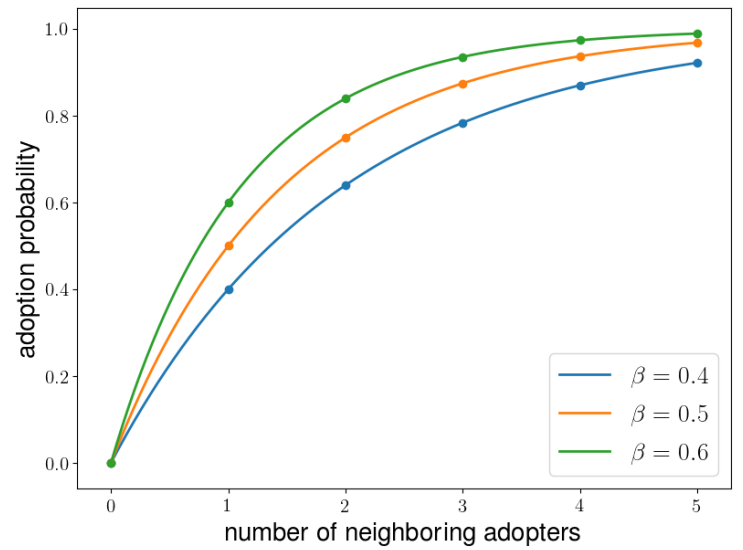

(A) Simple Activation Functions

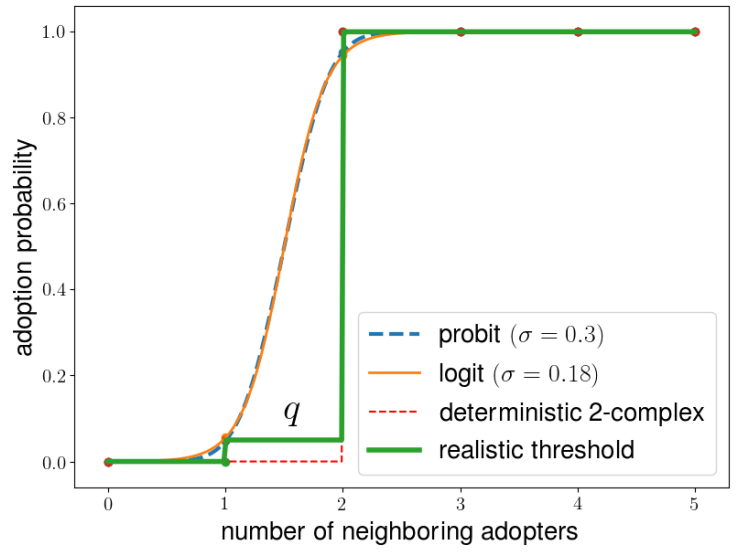

(B) Complex Activation Functions

Fig. 1. Activation functions for $(A)$ simple contagion and $(B)$ variations on complex contagion. In the case of a simple activation function (A), every edge has an independent probability $\beta$ of transmitting infections (adoptions); subsequently, the probability of adoption with $x$ adopters in the social neighborhood is given by $1-(1-\beta)^{x}$. In the case of a realistic threshold-based contagion model (B), there is a non-zero probability $(q>0)$ of adoptions below threshold. In a probit activation function the probability of adoption with $x$ adopters in the social neighborhood is equal to $\Phi_{\theta, \sigma}(x)$, where $\Phi_{\theta, \sigma}(\cdot)$ is the normal CDF with mean $\theta$ and standard deviation $\sigma$. The logit activation function is given by $\Psi_{\theta, \sigma}(x)=\left(1+e^{(\theta-x) / \sigma}\right)^{-1}$. Both functions describe a noisy threshold response that converges to a deterministic threshold $\theta$ as $\sigma \rightarrow 0$.

\section{Results}

Consider the cycle-power- $k$ graph $\left(\mathcal{C}_{k}\right)$. Starting from a pair of adjacent $\left(\mathcal{C}_{1}\right.$-neighboring) infected nodes, it takes $n / k$ time steps for all the $n$ nodes on $\mathcal{C}_{k}$ to get infected via pure 2 -complex contagion. We examine the spreading speed of contagion as cycle edges (short ties) are removed and replaced with random edges (long ties). To this end, we first analyze the spread of contagion on the union graph $\mathcal{C}_{k} \cup \mathcal{G}_{n, p_{n}}$, where $\mathcal{G}_{n, p_{n}}$ is the Erdös-Rényi random graph model with edge probability $p_{n}$. We note that the union graph has $n$ nodes and the set of edges is the union of edges in the two graphs. Setting $p_{n}=(D-2 k) / n$ ensures that the expected degree of each node is kept fixed at $D$. Thus by varying $k$, we can study the spreading rate of contagion as the cycle edges are rewired and replaced by (random) long ties.

Theorem 1 derives the asymptotic rate of spread for 2-complex contagion on $\mathcal{C}_{2} \cup \mathcal{G}_{n, c / n}$. Our result immediately implies that the rate is faster than on $\mathcal{C}_{2+c / 2}$, thus emphasizing the usefulness of long ties in speeding up complex contagion as long as the required local structure (the cycle $\mathcal{C}_{2}$ ) is intact, even in the absence of any below-threshold adoption.

Theorems 2 and 3 study the rate of spread of complex contagion as the edges of $\mathcal{C}_{2}$ are rewired; this alters the short tie structure that is necessary for the local diffusion of complex contagion. We allow for a small probability $q_{n}$ of adoptions below threshold (i.e., simple adoptions). Formally, this implies that a node with at least one infected neighbor gets infected independently in each round with probability $q_{n}$. We rewire the $\mathcal{C}_{2} \backslash \mathcal{C}_{1}$ edges, keeping the average degree constant (equal to four). This interpolates between $\mathcal{C}_{2}$ and $\mathcal{C}_{1} \cup \mathcal{G}_{n, 2 / n}$ by rewiring the edges on $\mathcal{C}_{2} \backslash \mathcal{C}_{1}$. Theorem 2 establishes that if the probability of sub-threshold 

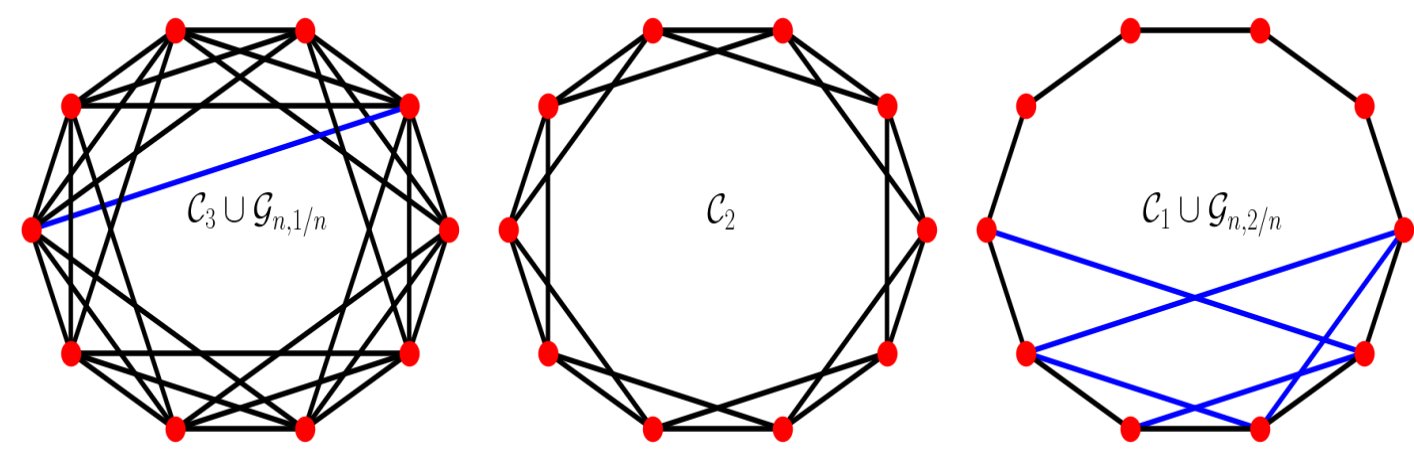

Fig. 2. Unions of cycle-graphs and random graphs. The cycle edge are colored black and random (long) edges are colored blue. The cycle-power- $k$ graph, denoted by $\mathcal{C}_{k}$, is constructed by connecting each node on an $n$-cycle to all nodes within its $k$-hop distance (i.e. the $2 k$ nearest neighbors), where the hop distance is measured on the cycle $\mathcal{C}_{1}$.

adoptions is large enough $\left(\sqrt{n} q_{n} \rightarrow \infty\right)$, the contagion spreads faster in $\mathcal{C}_{1} \cup \mathcal{G}_{n, 2 / n}$, compared to $\mathcal{C}_{2}$. Theorem 3 provides a more detailed picture, and analyzes the spreading time at any point on the interpolation path between $\mathcal{C}_{2}$ and $\mathcal{C}_{1} \cup \mathcal{G}_{n, 2 / n}$. At first, as we rewire $o(\sqrt{n})$ edges, the time to spread increases. In this scenario, there are too few long ties to facilitate complex contagion to faraway nodes, and the missing double edges incur a waiting time, thus slowing down the total time required. However, once $\omega(\sqrt{n})$ edges have been rewired, complex contagion spreads along the long ties. If $\sqrt{n} q_{n} \rightarrow \infty$, complex contagion via long ties facilitates the spread of contagion to the whole graph in $o(n)$ time. Note that this is much faster than the original spreading time over $\mathcal{C}_{2}$.

Before stating our results formally, we identify some of the mechanisms that govern the spreading rate of the contagion over $\mathcal{C}_{k} \cup \mathcal{G}_{n, p_{n}}$ graphs. The contagion is initialized by two infected neighbors on $\mathcal{C}_{1}$. The contagion spreads through two distinct sub-processes: (i) spreading along the cycle $\mathcal{C}_{1}$ via simple and complex contagion, and (ii) complex contagion along the long ties. The latter occurs when an uninfected node has at least two long ties connecting it to infected nodes. Initially, the infection spreads along the cycle. Once the infected nodes form intervals of sufficient length, the infection can spread to far away parts of the graph using the long ties. All our results hold with high probability (w.h.p.), i.e., with probability converging to one as the number of nodes $n \rightarrow \infty$.

Speed of Complex Contagion with Long Ties. In this section, we provide the asymptotic rate of 2 -complex contagion over $\mathcal{C}_{2} \cup \mathcal{G}_{n, c / n}$, and compare it with the spread over $\mathcal{C}_{2+c / 2}$. Complex contagion in the latter case, takes $n /(2+c / 2)=\Theta(n)$ time steps to spread. Our analysis below establishes that the diffusion time in $\mathcal{C}_{2} \cup \mathcal{G}_{n, c / n}$ is $O^{*}\left(n^{2 / 3}\right)$, significantly faster than $\Theta(n)$. In fact, we show that the spreading time is w.h.p. greater than $o\left(n^{2 / 3}\right)$, thus essentially fixing the order of the spreading time at $n^{2 / 3}$ (up to some logarithmic factors).

Our result emphasizes the intuitive understanding that rewiring accelerates the spread of complex contagion (from $\Theta(n)$ in $\mathcal{C}_{2+c / 2}$ to $O^{*}\left(n^{2 / 3}\right)$ in $\mathcal{C}_{2} \cup \mathcal{G}_{n, c / n}$ ), as long as the essential short tie structure (in our case $\mathcal{C}_{2}$ ) that facilitates local reinforcements, remains intact. 

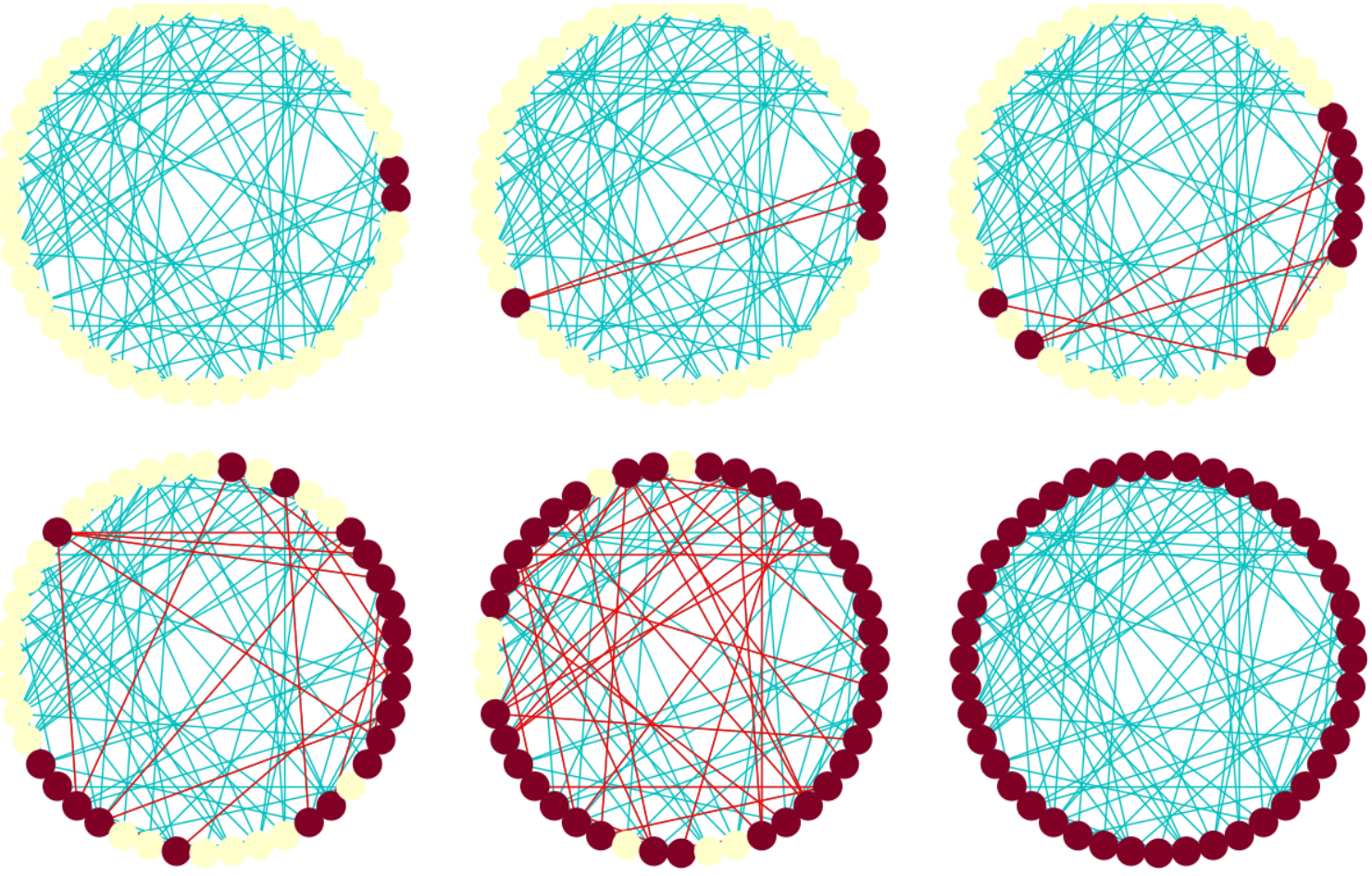

Fig. 3. Illustration of spreading via short and long ties. Each node is connected to its four nearest neighbors on the cycle $\left(\mathcal{C}_{2}\right)$ and, in addition, has two random (long) ties. The contagion spreads locally via the short ties of $\mathcal{C}_{2}$. Then there are multiple ties from the infected interval to a distant node, resulting in the contagion jumping across the cycle. The red edges in the figure are between the existing and newly infected nodes, highlighting the "path" of the spread. When two edges from an infected interval land on the same "faraway" node, they form a "wide-enough" bridge for the complex contagion to cross.

Theorem 1 (Rate of 2-complex contagion over $\mathcal{C}_{2} \cup \mathcal{G}_{n, c / n}$ ). The time to total spread in $\mathcal{C}_{2} \cup \mathcal{G}_{n, c / n}$ can be upper and lower bounded as follows:

(i) The entire graph is infected in time $2 n^{2 / 3}(\log \log n)^{2}$, w.h.p.

(ii) For any $\varepsilon>0$, the number of nodes infected by time $n^{2 / 3-\varepsilon}$ is at most $n^{2 / 3}$ w.h.p.

In (31) the authors bound the spreading time of 2-complex contagion in the related NewmanWatts random graph model (43) by $\Omega(\sqrt{n / \log n})$ and $O\left(\sqrt[5]{n^{4} \log n}\right)$. Our results suggest that the order of the spreading time can be characterized more precisely as $O^{*}\left(n^{2 / 3}\right)$.

Figure 4 shows the time that it takes for the contagion to spread over $\mathcal{C}_{k} \cup \mathcal{G}_{n,(D-2 k) / n}$ graphs. The expected degree is fixed at $D$, which is set to $D=15$ in Figure 4A. Simulation results show that rewiring the cycle edges and replacing them with random long ties speeds up the spread of contagion. However, this trend is not carried through all the way until $k=2$. Note that if we rewire the cycle edges beyond $\mathcal{C}_{2}$, complex contagion may not spread to the entire graph. However, if there is a vanishing probability $\left(q_{n} \rightarrow 0\right)$ of below-threshold adoptions, even only along the cycle edges, the contagion can continue to spread. Our subsequent results in Theorems 2 and 3 address this case. 


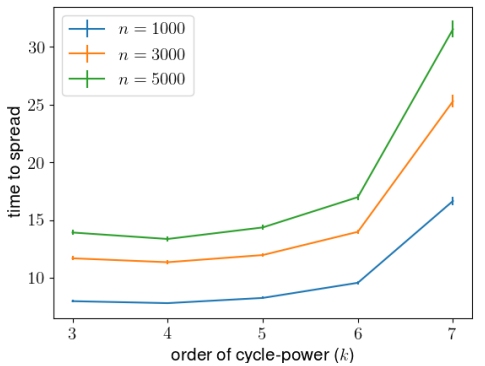

(A) $\mathcal{C}_{k} \cup \mathcal{G}_{n, p_{n}}, p_{n}=(15-2 k) / n$

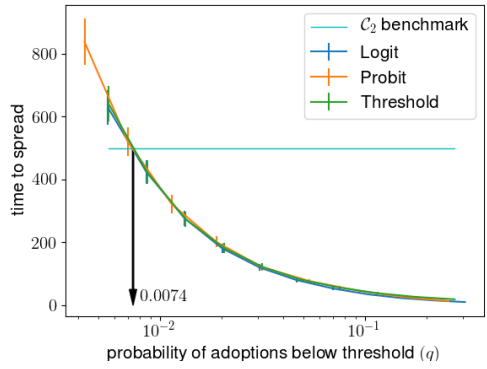

(B) $\mathcal{C}_{1} \cup \mathcal{G}_{n, 2 / n}, n=1000$

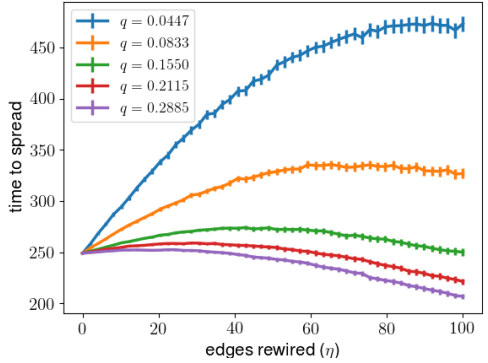

(C) $\mathcal{C}_{2}^{\eta}, n=500$

Fig. 4. Spreading time of complex contagion over cycle-power union random graphs with a fixed overall expected degree $D$, (A) $D=15$, (B) $D=4$ and (C) $D=4$. The spreading model in (A) is the 2-complex contagion (with no simple/sub-threshold adoptions, as in Theorem 1). In (B), we consider 2-complex contagion but now allow a simple adoption probability $q$ on every edge. In (C), we follow the same model as in Theorem 3: 2-complex contagion with sub-threshold adoption probability $q$ only along the $\mathcal{C}_{1}$ cycle edges. Each point is the average of 100, 500, and 1000 random draws for (A), (B), and (C), respectively. The vertical bars indicate the $95 \%$ normal confidence intervals around the means.

Speed of Complex Contagion with Simple Adoptions. We introduce and study a model where in addition to complex contagion we have a small amount of below-threshold adoption via the $\mathcal{C}_{1}$ edges, i.e. contagion can spread with (small) probability $q:=q_{n}$ along $\mathcal{C}_{1}$. In this setup, we compare the diffusion speed between $\mathcal{C}_{1} \cup \mathcal{G}_{n, 2 / n}$ and $\mathcal{C}_{2}$. We conclude that for large enough $q$ (having $\sqrt{n} q \rightarrow \infty$ turns out to be enough), contagion spreads faster in $\mathcal{C}_{1} \cup \mathcal{G}_{n, 2 / n}$ compared to $\mathcal{C}_{2}$. Hence, rewiring all the $\mathcal{C}_{2}$ edges but keeping the $\mathcal{C}_{1}$ edges intact, will speed up the spread of complex contagions, provided that there is a sufficient (but vanishing with increasing $n)$ probability of "simple" adoptions along the cycle-edges. The next theorem establishes that the rate of 2-complex contagion (with $q$ simple adoptions) over $\mathcal{C}_{1} \cup \mathcal{G}_{n, 2 / n}$ is of order $\sqrt{n} / q$. Formally, we establish the following result.

Theorem 2 (Rate of 2-complex contagion over $\mathcal{C}_{1} \cup \mathcal{G}_{n, 2 / n}$ with simple adoptions probability q). Allowing for simple adoptions to occur with probability $q$ along $\mathcal{C}_{1}$, the time to total spread in $\mathcal{C}_{1} \cup \mathcal{G}_{n, 2 / n}$ can be upper and lower bounded as follows:

(i) The entire graph is infected by time $\frac{4 \sqrt{n}}{q}(\log \log n)^{2}$ w.h.p.

(ii) For any $\varepsilon>0$, up to time $n^{1 / 2-\varepsilon} / q$, the number of infected nodes is at most $\sqrt{n}$ w.h.p.

Figure $4 \mathrm{~B}$ shows the spreading rate of contagion over $\mathcal{C}_{1} \cup \mathcal{G}_{n, 2 / n}$ with $n=1000$. We use three activation functions: logit, probit, and modified threshold with non-zero probability $(q)$ of adoptions below threshold. Indeed, logit and probit functions even allow for spontaneous adoptions (when there are no infected neighbors). Moreover, probability of adoptions above threshold is high but less than one. The simulation results show that spread of contagion over $\mathcal{C}_{1} \cup \mathcal{G}_{n, 1 / n}$ is faster than $\mathcal{C}_{2}$ when adoptions below threshold happen with probabilities greater than 0.0074. This is consistent with the predictions of Theorem 2, whereby for $\sqrt{n} q \rightarrow \infty$ the time to spread is $O^{*}(\sqrt{n} / q)=o(n)$ which is strictly faster than $n / 2$, the spreading time for complex contagion over $\mathcal{C}_{2}$. 

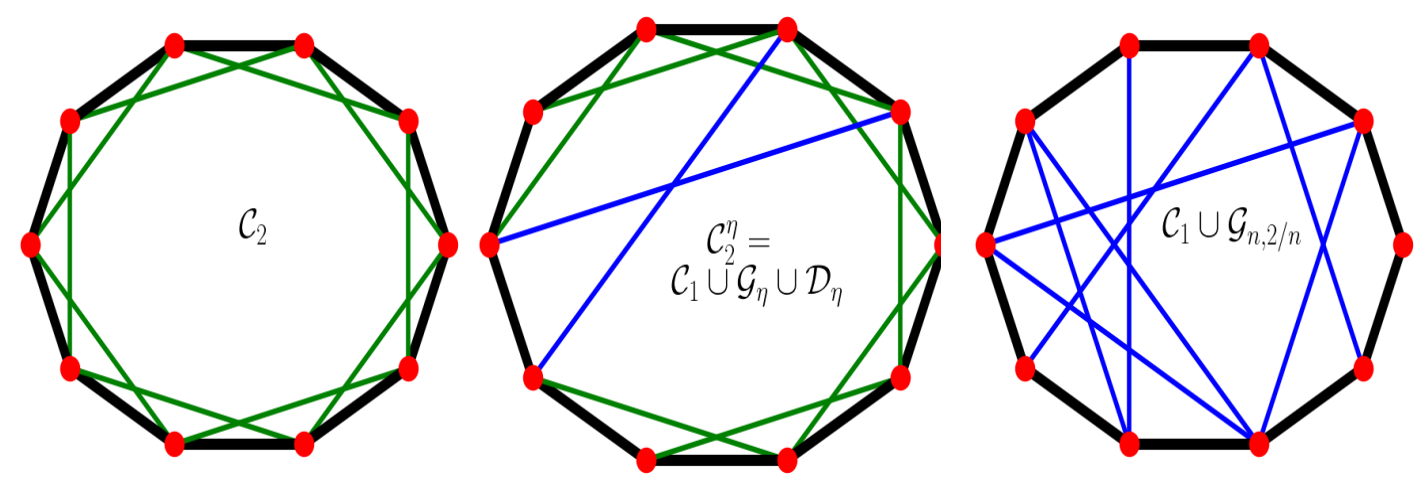

Fig. 5. In Theorems 2 and 3, we characterize the speed of spread as $\mathcal{C}_{2} \backslash \mathcal{C}_{1}$ edges (in green) are removed and replaced by random edges (in blue), allowing for simple contagions only along the edges of $\mathcal{C}_{1}$ (in black).

Speed of Complex Contagion with a Few Edges Rewired. Here we interpolate continuously between the random graphs $\mathcal{C}_{2}$ and $\mathcal{C}_{1} \cup \mathcal{G}_{n, \frac{2}{n}}$. We track the evolution of the spreading time along the interpolation path. Formally, we consider the following continuous time model. Define two random graph processes $\mathcal{D}_{\eta}$ and $\mathcal{G}_{\eta}$ that are coupled through the common index $\eta \geq 0$. The coupling is achieved through independent exponential variables that are associated with the edges of the graphs:

- Consider any pair of nodes $i, j \in[n]$. We associate an exponential random variable $X_{i j}>0$ with mean $n^{2}$ to each such pair. Given $\eta$, we include edge $i, j$ in random graph $\mathcal{G}_{\eta}$ if $X_{i j}<\eta$. Therefore, the random graph $\mathcal{G}_{\eta}$ is distributed as Erdös-Rényi with edge probability $\mathbb{P}\left\{X_{i j}>\eta\right\}=1-e^{-\eta / n^{2}}$.

- Similarly, with every edge $i, j$ in $\mathcal{C}_{2} \backslash \mathcal{C}_{1}$, we associate an exponential variable $Y_{i j}$ with mean $2 n$. For each $\eta$, edge $i, j$ is retained in $\mathcal{D}_{\eta}$ if $Y_{i, j}>\eta$. Therefore, for each $\eta$ the probability that the cycle edge $i, j$ is removed is $1-e^{-\eta / 2 n}$.

Consider the graph $\mathcal{C}_{1} \cup \mathcal{G}_{\eta} \cup \mathcal{D}_{\eta}$ in the regime $\eta=o(n)$. Note that for $\eta=o(n)$, the expected degree of nodes in $\mathcal{C}_{1} \cup \mathcal{G}_{\eta} \cup \mathcal{D}_{\eta}$ is $4+o(\eta / n)$. Hence, for $\eta=o(n)$ the average degrees of nodes in $\mathcal{C}_{1} \cup \mathcal{G}_{\eta} \cup \mathcal{D}_{\eta}$ remains fixed at four, which is the degree of nodes in $\mathcal{C}_{2}$. Motivated by this observation, we refer to $\mathcal{C}_{1} \cup \mathcal{G}_{\eta} \cup \mathcal{D}_{\eta}$ as the " $\eta$-rewired $\mathcal{C}_{2}$ " random graph and denote it by $\mathcal{C}_{2}^{\eta}$. We will use $\mathcal{C}_{2}^{\eta}$ to study what happens as we rewire the $\mathcal{C}_{2} \backslash \mathcal{C}_{1}$ edges. In this context, $\eta$ denotes the "expected" number of edges that are rewired to construct the random graph $\mathcal{C}_{2}^{\eta}$ from $\mathcal{C}_{2}$.

In the subsequent discussion, we parameterize $\eta=n^{\delta}$ for $\delta \in(0,1)$ and study the speed of infection $\mathcal{C}_{2}^{\eta}$ in two regimes:

(i) For $\delta \in\left(0, \frac{1}{2}\right)$ we show that the spreading slows down with increasing $\delta$. Hence, rewiring is detrimental to the spread of contagion. In this regime, it is unlikely that two random edges land on the same "faraway" node. Therefore, complex contagion cannot yet reliably spread through the long ties when $\delta<1 / 2$. In the language of Figure 3, the long ties are yet too few to for wide-enough bridges that facilitate the spread of complex contagion. Under such circumstances, the rewiring only slows down the spread, since it introduces new break points for the spread of complex contagion along the (short) cycle edges. 
(ii) For $\delta \in\left(\frac{1}{2}, 1\right)$ we show that contagion takes order $n^{3 / 2-\delta}+\sqrt{n} / q$ time to spread. For $\delta$ large enough, $\sqrt{n} / q$ is the dominant term that fixes the spreading speed. However, for $q=n^{-1 / 2+\delta^{\prime}} \gg 1 / \sqrt{n}$ we can specify a range of $\delta$ for which increasing $\delta$ increases the speed, with complex contagion spreading through the long ties. In particular, if $q=n^{-1 / 2+\delta^{\prime}}$, then for $\frac{1}{2}<\delta<\frac{1}{2}+\delta^{\prime}$ contagion spreads faster in the $\eta$-rewired $\mathcal{C}_{2}$.

The following theorem formalizes these intuitions.

Theorem 3 (Rate of 2-complex contagion over $\mathcal{C}_{2}^{\eta}$ with simple adoptions probability $q$ ). Let $\delta \in(0,1)$, and consider $\eta=n^{\delta}$ :

(i) For fixed $\delta \in\left(0, \frac{1}{2}\right)$, w.h.p., the total spread time is at least $\frac{n}{2}+\frac{\eta}{4 q}$.

(ii) For fixed $\delta \in\left(\frac{1}{2}, 1\right)$, the time to global spread can be upper and lower bounded as follows:

(a) The entire graph is infected by time $4\left(\sqrt{n} / q+n^{3 / 2-\delta}\right)(\log \log n)^{2}$ w.h.p.

(b) For any $\varepsilon>0$, the number of infected nodes by time $n^{1 / 2-\varepsilon} / q$ is at most $\sqrt{n}$ w.h.p.

In comparison, the time for infection on $\mathcal{C}_{2}$ is exactly $n / 2$. Thus the first part establishes that for $\eta=o(\sqrt{n})$, the infection spread is slowed down due to the missing edges along the cycle.

Figure $4 \mathrm{C}$ shows the spreading time versus the rewiring parameters $\eta$ for the $\mathcal{C}_{2}^{\eta}$ random graph. It confirms that when the probability of adoptions below threshold $q$ is large enough, the spread of contagions speeds up with the increasing rewiring of the $\mathcal{C}_{2} \backslash \mathcal{C}_{1}$ cycle edges. The plots further verify the theoretical predictions of Theorem 3 about an initial slow down followed by the speeding up of the contagion process as more cycle edges are rewired. In the SI Appendix, Fig. S2, we include additional simulation results with models that allow for all edges (not just $\mathcal{C}_{1}$ ) to have simple contagion probability $q$. The additional results demonstrate the same type of qualitative behavior in that rewiring accelerates the spread of complex contagion for large enough $q$. However, the initial slow down phase that is predicted by Theorem 3 and observed in Figure $4 \mathrm{C}$ is not present in Figure S2, where we allow all edges to have simple contagion probability $q$. Indeed, the slow down for $\eta=o(\sqrt{n})$ is an consequence of the handicap that we impose on our model: allowing simple contagion only along the $\mathcal{C}_{1}$ edges. Under this restriction, long ties can facilitate the spread only if they form wide-enough bridges for complex contagion to pass (see Figure 3). If the number of rewired edges is too few, $\eta=o(\sqrt{n})$, then the probability that two edges from an infected "island" land on the same faraway node is very small: the "bridges" are too narrow for complex contagion to pass. Allowing simple contagion along all edges (as in Figure S2) will only make our claim stronger: rewiring accelerates realistic complex contagions even further if we allow simple contagion along the rewired edges. Our simulations in the next section with other modeling variations, and over empirical network data, support the robustness of this claim. 


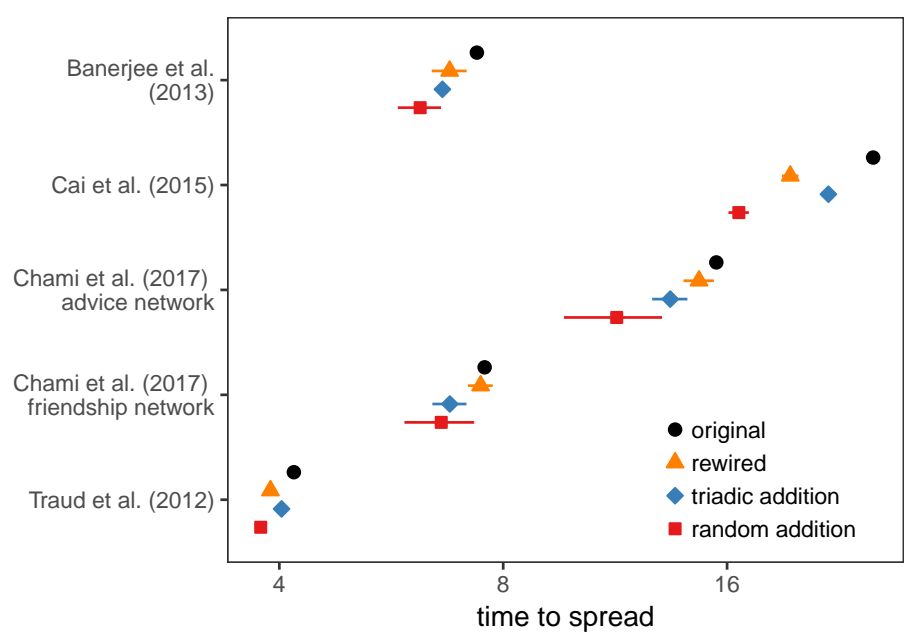

(A) Mean Spread Times

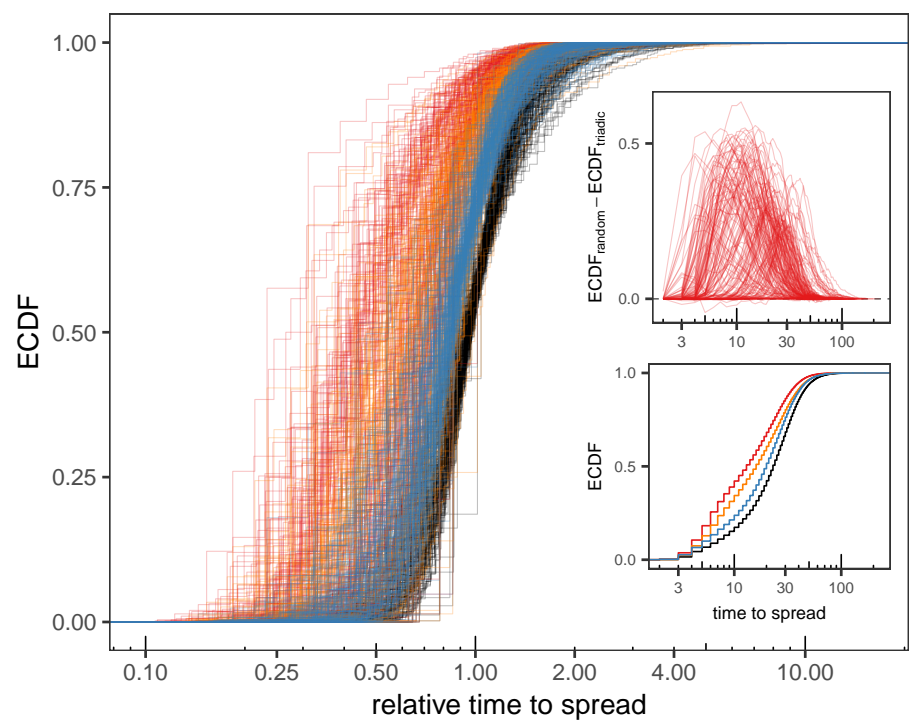

(B) Empirical Cumulative Distribution Functions

Fig. 6. (A) Mean time to spread in each set of empirical networks. Each point averages over all networks in that set. Error bars are $95 \%$ confidence intervals for the difference from the original network computed by treating each network as a single observation. (B) Distribution of time to spread for each of the 175 networks of Chinese households in Cai et al. (44). For each village, we plot the empirical cumulative distribution function (ECDF) of the spreading times in the original village (black) and under rewiring (orange), as well as random (red) and triad-closing (blue) edge additions. Hence, the main figure overlays $4 \times 175=700$ curves, corresponding to the ECDFs of the 500 spreading time samples computed for each village under the four conditions. Time to spread is normalized by the mean time to spread in the original network. Compared with closing triads, adding random edges consistently speeds up spread, as illustrated by the positive difference in ECDFs (upper inset). The positive difference in each case implies stochastic dominance: the spreading time over the village network with $10 \%$ added triad-closing edges dominates (is slower than) the spreading time over the network with the $10 \%$ new edges added randomly. The distributions of time to spread averaging over all 175 networks (lower inset) illustrate that both rewiring and random additions speed up the contagion. 
Simulations with Empirical Networks. We use five sets of empirical social networks; see Materials and Methods for a description of each. For each social network, contagion begins from two adjacent random seeds, and we measure the time to $90 \%$ spread under four conditions: (i) the original networks (no intervention), (ii) with $10 \%$ of edges rewired, (iii) with $10 \%$ added edges selected proportional to the number of triads they close, and (iv) with $10 \%$ new edges added randomly. For each intervention type, we simulate the spread times over the modified networks 500 times. In these simulations, a node adopts with certainty if it has at least two adopter neighbors; moreover, we fix the probability of adoption with a single neighboring adopter at $q=0.05$. Across all five sets of networks, random rewiring decreases mean time to spread (Figure 6A). Furthermore, adding random, rather than triad-closing, edges likewise reduces mean time to spread. We further examine spreading times for each network in the largest set - households in 175 villages in rural China (44) - in Figure 6B, where we observe a corresponding shift in the distribution of spreading times.

These results are robust to a number of variations; see SI Appendix, section S5. There we present a variation of this model where the probability of adoptions above threshold (called $\rho)$ is less than one $(\rho=0.5, q=0.025)$, as well as a case with very small simple adoption probability $(\rho=1, q=0.001)$. In another variation, infected nodes transition to an inactive state (with probability $\gamma=0.5$ ), in which they are no longer infectious, although they are still counted as being infected (adopters). In yet another variation, we consider a fractional threshold model with relative thresholds set to $\theta^{\star}=0.5$. Simulation results in all cases reveal the same direction for the effect of interventions, although the effect sizes vary.

Our simulations indicate that in many real social networks rewiring the edges causes these contagions to spread faster. Moreover, contagion spreads faster when new edges are added uniformly at random rather than with probability proportional to the number of open triads that they close. The latter suggests that it is advantageous to introduce new ties that close fewer triads. This is true even if the decisions to adopt rely on local reinforcement from the neighboring adopters (for example, with $\rho=1$ and $q=0.001$ ).

\section{Discussion}

Contrary to the ideas surrounding the "weakness of long ties" $(23,24,27)$, we find that interventions that introduce long ties via random rewiring or adding random ties accelerate the spread of complex contagions. In realistic versions of such contagions, there is at least small probability for adoption to occur even when there is only a single adopter in the social neighborhood. This is enough to change the landscape of results, thereby leading to the conclusion that long ties accelerate these contagions - just as they do for simple contagions.

We studied 2-complex contagion in a handicapped model where below-threshold adoptions happen only along "short" cycle edges. Below-threshold adoption rates in the order as low as $1 / \sqrt{n}$ are enough to induce a faster spread over a rewired network compared to the original structure. Our analysis indicates that the rate of spread over cycle union random graph structures is determined by the time that it takes for the infected intervals along the cycle to grow long enough, to makes the spread of complex contagion through their long ties a probable event. This idea is reasonably general and can be applied to modeling variations where above-threshold adoption occurs with a probability less than one $(\rho<1)$ or the adoption thresholds are greater than two $(\theta>2)$. In both cases the spread will be slowed down either 
to wait for above-threshold adoption to occur at the slower $1 / \rho$ rate or for the intervals to grow longer to make a more stringent $\theta$-complex adoption probable.

We test the generality of these results using simulations with empirical networks subject to multiple structural interventions. Here long ties again accelerate the spread of complex contagions, whether operationalized as rewiring the observed network or adding random, rather than triad-closing, edges.

Our results indicate that introduction of long ties are more effective for accelerating the spread of social contagion - whether simple and complex. Thus, we propose a more unified recommendation for structural interventions by inclusion of long ties in less clustered neighborhoods to cause a faster spread. This conclusion is consistent with empirical studies that identify structural diversity as an indicator of increased adoption (28) and document the prevalence of long ties with high information-exchange bandwidth (45).

However, interventions in social networks are often unable to directly form arbitrary relationships; rather they typically consist in some encouragement to interaction. For example, individuals can be randomly assigned to groups, but only some endogenously form friendships, with substantial consequence for the success of such interventions (11). While there can be noncompliance in edge formation, our simulations suggest that even if one can induce triad-closing edges to form at a greater rate, focusing on forming long ties could still be more effective. In the networks of households in rural China (44), we observe that even with $25 \%$ additional short, triad-closing ties, spread is slower than with only $10 \%$ additional long, random ties (SI Appendix, Figure S9). Nonetheless, our results do not address the decision to form a tie or how the latter is correlated with the subsequent adoption decisions. Rather, we clarify the effect that the introduction of new ties has on the speed of spread. We propose the confluence of these two decisions - whether to form a tie, perhaps in response to an intervention, and whether to adopt a behavior given its adoption by network neighbors - as a topic for further study.

\section{Materials and Methods}

The empirical network data for our simulation studies are derived from publicly available data $(8,44,46,47)$. The Cai et al. (44) data is comprised of 175 social networks of Chinese farm villages that are collected in the study of farmers being encouraged to sign up for a weather insurance product. The friendship and health advice network data are collected by Chami et al. (8) from 17 rural villages in Uganda. The Banerjee et al. (46) data contains the interconnection data for multi-dimensional social relations in 77 villages in southern India. Traud et al. (47) data contains the Facebook friendship networks at U.S. colleges and universities; we use the 40 smallest networks, for which such simulations are more computationally practical. The village networks in the first three sets have as few as tens of nodes but have typically hundreds of nodes. A typical Facebook college network has thousands of nodes. Table S1, in SI Appendix, summarizes the statistics for each set of networks. Code for reported simulations can be accessed from https://github.com/aminrahimian/social-contagion/wiki.

ACKNOWLEDGMENTS. The authors would like to thank Colin Sandon for pointing out an error in the earlier version of Theorem 1. E.M. is partially supported by NSF grant CCF 1665252, DOD ONR grant N00014-17-1-2598, and NSF grant DMS-1737944. 
1. Leskovec J, Adamic LA, Huberman BA (2007) The dynamics of viral marketing. ACM Transactions on the Web (TWEB) 1(1):5.

2. Kempe D, Kleinberg J, Tardos É (2003) Maximizing the spread of influence through a social network in Proceedings of the ninth ACM SIGKDD international conference on Knowledge discovery and data mining. (ACM), pp. 137-146.

3. Hinz O, Skiera B, Barrot C, Becker JU (2011) Seeding strategies for viral marketing: An empirical comparison. Journal of Marketing 75(6):55-71.

4. Libai B, Muller E, Peres R (2013) Decomposing the value of word-of-mouth seeding programs: Acceleration versus expansion. Journal of Marketing Research 50(2):161-176.

5. Beaman L, BenYishay A, Magruder J, Mobarak AM (2018) Can network theory-based targeting increase technology adoption?, (National Bureau of Economic Research), Technical Report 24912.

6. Cohen R, Havlin S, Ben-Avraham D (2003) Efficient immunization strategies for computer networks and populations. Physical Review Letters 91(24):247901.

7. Preciado VM, Zargham M, Enyioha C, Jadbabaie A, Pappas GJ (2014) Optimal resource allocation for network protection against spreading processes. IEEE Transactions on Control of Network Systems 1(1):99-108.

8. Chami GF, Ahnert SE, Kabatereine NB, Tukahebwa EM (2017) Social network fragmentation and community health. Proceedings of the National Academy of Sciences 114(36):E7425-E7431.

9. Chaoji V, Ranu S, Rastogi R, Bhatt R (2012) Recommendations to boost content spread in social networks in Proceedings of the 21st international conference on World Wide Web. (ACM), pp. 529-538.

10. Valente TW (2012) Network interventions. Science 337(6090):49-53.

11. Carrell SE, Sacerdote BI, West JE (2013) From natural variation to optimal policy? The importance of endogenous peer group formation. Econometrica 81(3):855-882.

12. Cerdeiro DA, Dziubiński M, Goyal S (2017) Individual security, contagion, and network design. Journal of Economic Theory 170:182-226.

13. Dodds PS, Watts DJ (2005) A generalized model of social and biological contagion. Journal of Theoretical Biology 232(4):587-604.

14. Watts DJ, Strogatz SH (1998) Collective dynamics of 'small-world' networks. Nature 393(6684):440.

15. Hébert-Dufresne L, Noël PA, Marceau V, Allard A, Dubé LJ (2010) Propagation dynamics on networks featuring complex topologies. Physical Review E 82(3):036115.

16. Granovetter MS (1973) The strength of weak ties. American Journal of Sociology 78(6):1360-1380.

17. Gee LK, Jones JJ, Fariss CJ, Burke M, Fowler JH (2017) The paradox of weak ties in 55 countries. Journal of Economic Behavior \& Organization 133:362-372.

18. Galeotti A, Goyal S, Jackson MO, Vega-Redondo F, Yariv L (2010) Network games. The Review of Economic Studies 77(1):218-244.

19. Blume LE (1993) The statistical mechanics of strategic interaction. Games and Economic Behavior 5(3):387-424.

20. Morris S (2000) Contagion. The Review of Economic Studies 67(1):57-78.

21. Young HP (2011) The dynamics of social innovation. Proceedings of the National Academy of Sciences 108(Supplement 4):21285-21291.

22. Granovetter M (1978) Threshold models of collective behavior. American Journal of Sociology 83(6):1420-1443.

23. Centola D, Macy M (2007) Complex contagions and the weakness of long ties. American Journal of Sociology 113(3):702-734.

24. Montanari A, Saberi A (2010) The spread of innovations in social networks. Proceedings of the National Academy of Sciences 107(47):20196-20201.

25. Bakshy E, Rosenn I, Marlow C, Adamic L (2012) The role of social networks in information diffusion in Proceedings of the 21st international conference on World Wide Web. (ACM), pp. 519-528.

26. Bakshy E, Eckles D, Yan R, Rosenn I (2012) Social influence in social advertising: Evidence from field experiments in Proceedings of the 13th ACM conference on electronic commerce. (ACM), pp. 146-161.

27. Centola D (2010) The spread of behavior in an online social network experiment. Science 329(5996):1194-1197.

28. Ugander J, Backstrom L, Marlow C, Kleinberg J (2012) Structural diversity in social contagion. Proceedings of the National Academy of Sciences 109(16):5962-5966. 
29. Janson S, Kozma R, Ruszinkó M, Sokolov Y (2016) Bootstrap percolation on a random graph coupled with a lattice. Electronic Journal of Combinatorics.

30. Durrett R (2010) Some features of the spread of epidemics and information on a random graph. Proceedings of the National Academy of Sciences 107(10):4491-4498.

31. Ghasemiesfeh G, Ebrahimi R, Gao J (2013) Complex contagion and the weakness of long ties in social networks: revisited in Proceedings of the fourteenth ACM conference on Electronic Commerce. (ACM), pp. 507-524.

32. Ebrahimi R, Gao J, Ghasemiesfeh G, Schoenebeck G (2015) Complex contagions in Kleinberg's small world model in Proceedings of the 2015 Conference on Innovations in Theoretical Computer Science. (ACM), pp. 63-72.

33. Ebrahimi R, Gao J, Ghasemiesfeh G, Schoenbeck G (2017) How complex contagions spread quickly in preferential attachment models and other time-evolving networks. IEEE Transactions on Network Science and Engineering 4(4):201-214.

34. Schoenebeck G, Yu FY (2016) Complex contagions on configuration model graphs with a powerlaw degree distribution in International Conference on Web and Internet Economics. (Springer), pp. 459-472.

35. Nematzadeh A, Ferrara E, Flammini A, Ahn YY (2014) Optimal network modularity for information diffusion. Physical Review Letters 113(8):088701.

36. Jackson MO, Yariv L (2007) Diffusion of behavior and equilibrium properties in network games. American Economic Review 97(2):92-98.

37. Abrahamson E, Rosenkopf L (1997) Social network effects on the extent of innovation diffusion: $A$ computer simulation. Organization Science 8(3):289-309.

38. Newman ME, Watts DJ (1999) Renormalization group analysis of the small-world network model. Physics Letters A 263(4-6):341-346.

39. Balogh J, Pittel BG (2007) Bootstrap percolation on the random regular graph. Random Structures \& Algorithms 30(1-2):257-286.

40. Amini H, Fountoulakis N (2014) Bootstrap percolation in power-law random graphs. Journal of Statistical Physics 155(1):72-92.

41. Amini $H$ (2010) Bootstrap percolation and diffusion in random graphs with given vertex degrees. The Electronic Journal of Combinatorics 17(1):25.

42. Acemoglu D, Ozdaglar A, Yildiz E (2011) Diffusion of innovations in social networks in Decision and Control and European Control Conference (CDC-ECC), 2011 50th IEEE Conference on. (IEEE), pp. 2329-2334.

43. Newman ME, Watts DJ (1999) Scaling and percolation in the small-world network model. Physical Review E 60(6):7332.

44. Cai J, De Janvry A, Sadoulet E (2015) Social networks and the decision to insure. American Economic Journal: Applied Economics 7(2):81-108.

45. Park PS, Blumenstock JE, Macy MW (2018) The strength of long-range ties in population-scale social networks. Science 362(6421):1410-1413.

46. Banerjee A, Chandrasekhar AG, Duflo E, Jackson MO (2013) The diffusion of microfinance. Science 341(6144):1236498.

47. Traud AL, Mucha PJ, Porter MA (2012) Social structure of Facebook networks. Physica A: Statistical Mechanics and its Applications 391(16):4165-4180. 


\title{
Supplementary Information for
}

\section{Long ties accelerate noisy threshold-based contagions}

\author{
Dean Eckles, Elchanan Mossel, M. Amin Rahimian and Subhabrata Sen \\ E-mails:\{eckles,elmos,rahimian,ssen90\}@mit.edu
}

\section{This PDF file includes:}

Supplementary text

Figs. S1 to S9

Table S1

References for SI reference citations

\section{Supporting Information Text}

This Supplementary Information is organized in four sections that parallel the four parts of the Results section in the main text. Sections S2, S3, and S4 include the proofs for Theorems 1, 2 and 3 form the main text, as well as additional simulations. In Section S5, we expand on our study of empirical networks and present additional simulation results under various contagion models that deviate from those considered in the main text. These additional results support the robustness of our claims against modeling variations.

Notation: For convenience of the reader, we collect here some notation that will be used throughout in the subsequent discussion. For sequences of real numbers, we use the usual Bachman-Landau notation $O(\cdot)$, o(·) and $\Theta(\cdot)$. Further, for a sequence of non-negative real numbers $\left\{a_{n}: n \geq 1\right\}$ and a sequence of random variables $\left\{X_{n}: n \geq 1\right\}$, we say that $X_{n}=o\left(a_{n}\right)$ if $X_{n} / a_{n} \stackrel{P}{\rightarrow} 0$ as $n \rightarrow \infty$. Similarly, we declare $X_{n}=O\left(a_{n}\right)$ if there exists a universal constant $C>0$ such that $\mathbb{P}\left(\left|X_{n}\right| / a_{n} \leq C\right) \rightarrow 1$ as $n \rightarrow \infty$. Finally, say that $X_{n}=\Theta\left(a_{n}\right)$ if there exist universal constants $0<c<C<\infty$ such that $\mathbb{P}\left(c a_{n}<X_{n}<C a_{n}\right) \rightarrow 1$ as $n \rightarrow \infty$. For two random variables $X, Y$, we set $X \preceq Y$ if $X$ is stochastically dominated by $Y$. A sequence of events $\left\{A_{n}\right\}$ occurs with high probability as $n \rightarrow \infty$ (referred to as w.h.p.) if $\mathbb{P}\left(A_{n}^{c}\right) \rightarrow 0$ as $n \rightarrow \infty$.

\section{S1. Adoption probabilities by the number of adopting peers in real data}

We use a number of prior empirical studies to report on the relative rates of adoptions when there are $k$ and $k-1$ adopting neighbors: $p(k) / p(k-1)$, for integers $k>1$. We find that the reported ratios are less than five, consistently, across a multitude of studies (Figure S1); thus, there is evidence against homogeneous deterministic thresholds for adoption.

Aral et al. (2009) in (1) delineate how much of the clustering in the observed patterns of adoptions can be explained by contagion as opposed to homophily. The adoption patterns demonstrate significant clustering in terms of both network location of the adopters as well as the time at which neighboring nodes become adopters. However, the correlated outcomes can be attributed to both contagion and homophily: On the one hand, the linked nodes influence each other in their decisions to adopt; and on the other hand, they simply have greater likelihoods of displaying correlated outcomes as a consequence of their similar attributes. Using a dynamic matched sample estimation framework*, Aral et al. (2009) show that previous methods over-estimate peer influence in a study of the global instant messaging network of 27.4 million users, using data on the day-by-day adoption of a mobile service application called Yahoo Go mobile. In Fig. 3B (right inset) of (1), Aral et al. (2009) report the adoption ratios among the matched pairs versus the number of adopter friends. We have extracted and plotted these ratios in Figure S1.

In (2), Bakshy et al. (2012) present their results from a large-scale field experiment about sharing URLs on Facebook with 253 million subjects. The experiment randomizes whether or not individuals are exposed via Facebook to information about their friends' sharing behavior. The authors find that additional exposure has an increasing causal effect on the propensity to share. We extracted the probability of sharing versus the number of sharing friends

\footnotetext{
* They consider four different treatment levels corresponding to having one, two, three, or four adopter friends and estimate the treatment probabilities at different levels as a function of observable and latent node characteristics using logistic regression. They use the estimated treatment probabilities to create a dynamic matched sample of treated and untreated nodes over time: Every treated node (having one or more friends who are adopters) is matched with an untreated node (having fewer number of adopter friends) whose likelihoods of being treated (propensity scores) are closest.
} 
on the Facebook News Feed from Fig. 4(a) of (2). This is the observational association, as the experiment only randomizes exposure or non-exposure. We have plotted the ratio of probabilities for different values of $k$ in Figure S1.

In a controlled study to test for effects of complex contagion in (3), Centola (2010) randomizes subjects between two network conditions: a clustered lattice consisting entirely of short ties, and a random regular graph with very few triangle (short ties). The node degrees in both conditions are fixed and the same. The author studies the spread of a health-related behavior (registering for a health forum website) in an online community in which users were informed about the activities of their assigned neighbors ("health buddies") through email invitations to adopt the same behavior. The faster spread over the clustered lattice has been interpreted as evidence of complex contagion. In Figure S1, we have extracted the values reported in Figure 3 of (3), where up to three additional social signals significantly increases the probability of adoption.

Mønsted et al. (2017) in (4) present the results of a non-randomized field experiment conducted on the Twitter social network. They test the adoption of new hashtags using a network of Twitter bots ("botnet") with a large number of followers. The large user base who follow multiple bots are exposed to coordinated interventions by the bots to test the effects of multiple exposures. They observe that their proposed model of complex contagion is a better fit to the observed adoption rates than an alternative simple contagion model. We have extracted the percentage of retweets versus the total number of unique exposures from Figure 3A of (4) and plotted the results in Figure S1.

In (5), Ugander et al. (2012) use e-mail invitations for joining Facebook to study the growth of Facebook's user base. In a corpus of 54 million such email invitations, they analyze the probability of accepting an invitation as a function of the structure of the contact neighborhoods. They observe that adoption probabilities are better explained by the number of connected components in the contact neighborhoods; thus highlighting the effect of structural diversity in determining the conversion decisions. In Figure S1, we have plotted the ratios of the aggregate conversion rates for each neighborhood size. This data, provided by the authors, is presented in their Figure 1.

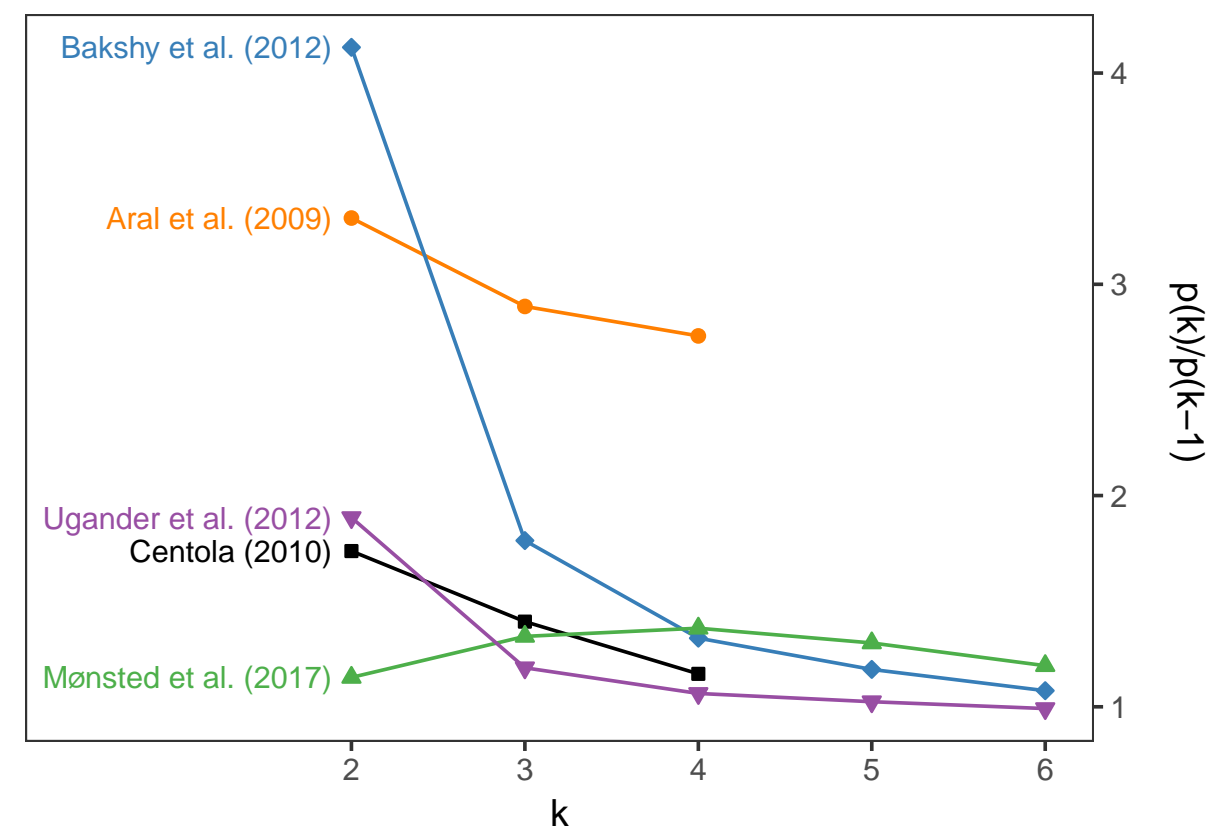

Fig. S1. Empirical adoption rate ratios

\section{S2. Spread of complex contagion over $\mathcal{C}_{2}$ union random graphs (Theorem 1)}

In this section we provide the asymptotic rate of 2-complex contagion over $\mathcal{C}_{2} \cup \mathcal{G}_{n, c / n}$, and compare it with $\mathcal{C}_{2+c / 2}$. We conclude that the diffusion speed is faster in the rewired graph. Therefore, we emphasize the understanding that rewiring facilitates the spread of complex contagion as long as the required structure for total spread $\left(\mathcal{C}_{2}\right)$ is intact.

\section{A. Spreading time lower-bound for complex contagion.}

Theorem S2.1 (Upper-Bounding the number of infected nodes until $t=o\left(n^{2 / 3}\right)$ ). Let $I_{t}$ denote the number of infected nodes at time $t$. For $t=o\left(n^{2 / 3}\right), I_{t}=o\left(n^{2 / 3}\right)$. 
Proof. Let $\mathcal{I}_{t}$ be the set of all infected nodes at time $t$ and let $\mathcal{B}_{t}$ denote the interval of length $2 t$ from the deterministic growth of the two neighboring initial seeds. Note that the vertices in $\mathcal{B}_{t}$ are always infected due to two-complex contagion along $\mathcal{C}_{2}$; hence, $B_{t}=\left|\mathcal{B}_{t}\right|=2 t=T$. We define $\mathcal{I}_{t}^{0}=\mathcal{B}_{t}$ and note that $\mathcal{I}_{t}^{0} \subset \mathcal{I}_{t}$. However, other vertices might be infected due to complex contagion along the edges of $\mathcal{G}_{n, c / n}$. To control these secondary infections, we introduce an algorithm which proceeds in rounds. These rounds are indexed by $\tau$ and they build up the infected set $\left\{\mathcal{I}_{t}^{\tau}: \tau \geq 1\right\}$ by exposing new random edges connecting infected vertices, identified at the current round, to "healthy"-vertices. The analysis below tracks the growth of the infected set over iterations, and establishes that for $t=o\left(n^{2 / 3}\right)$, the algorithm terminates after two rounds with high probability. Further, the additional infected vertices gained are $o\left(n^{2 / 3}\right)$ in number, and all isolated. Thus they do not give rise to secondary infections along $\mathcal{C}_{2}$ via 2-complex contagion.

Let us introduce the sequential algorithm formally before we proceed further. For notational convenience, let $\left\{\mathcal{S}_{t}^{\tau}: \tau \geq 1\right\}$ denote nodes with exactly one neighbor in $\mathcal{I}_{t}^{\tau}$ and call them "susceptible" nodes. Further, let $\mathcal{H}_{t}^{\tau}$ denote nodes that have no neighbors in $\mathcal{I}_{t}^{\tau}$ and call them "healthy" nodes. Moreover, we denote the nodes that are added to the infected set at round $\tau$ by $\mathcal{A}_{t}^{\tau}=\mathcal{I}_{t}^{\tau} \backslash \mathcal{I}_{t}^{\tau-1}$. Finally, for $v \in[n]$ and $C \subset[n], \mathcal{N}_{C}(v)$ will denote the number of neighbors of $v$ in the set $C$.

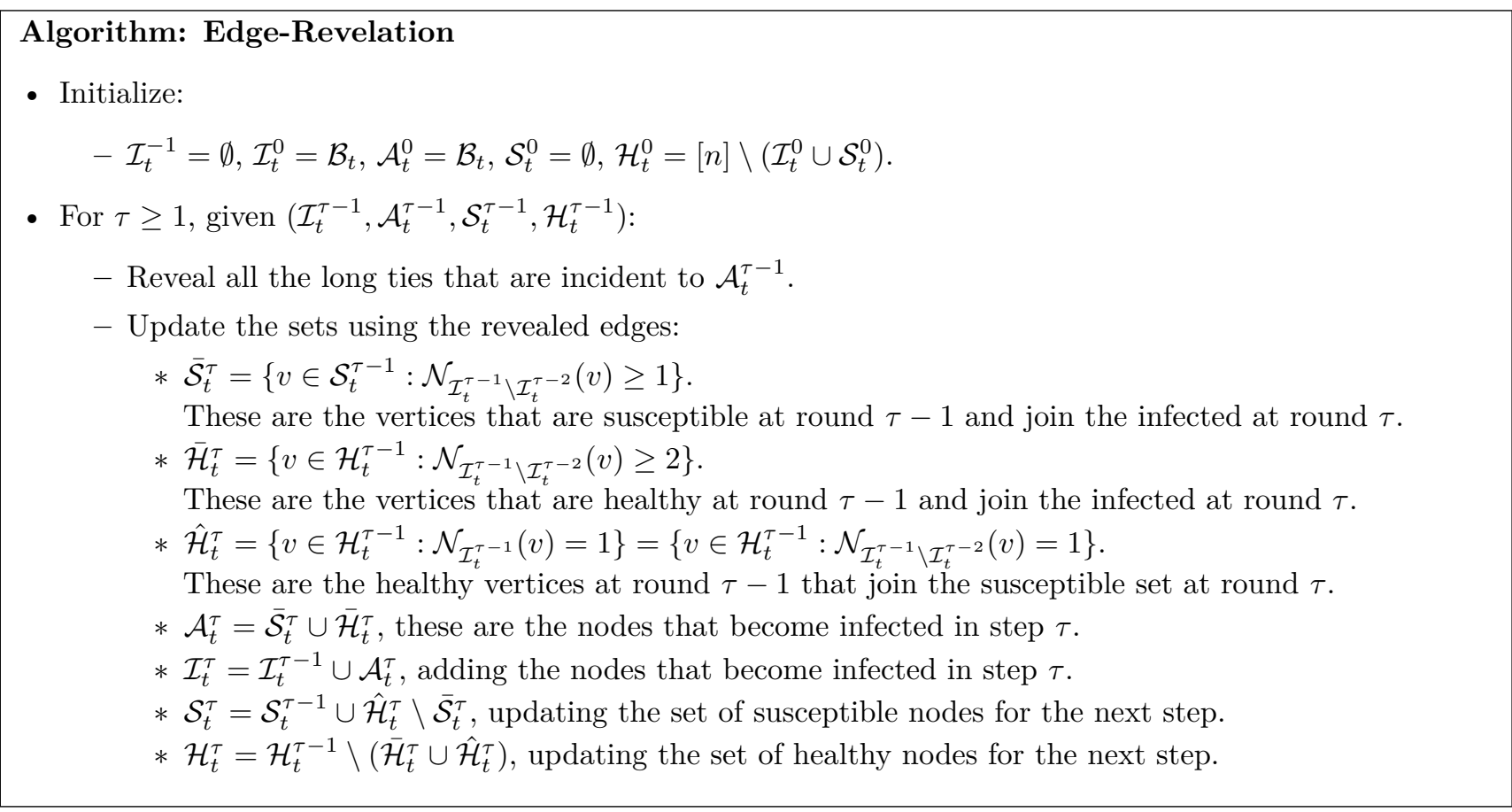

Recall that for notational convenience, we use roman fonts to refer to sizes of sets introduced in the Edge-Revelation algorithm - for example, $H_{t}^{0}=\left|\mathcal{H}_{t}^{0}\right|, B_{t}=\left|\mathcal{B}_{t}\right|$ and so on. We will refer to the natural filtration associated with the sequential procedure in the Edge-Revelation algorithm as $\left\{\mathcal{F}_{\tau}: \tau \geq 1\right\}$.

To track the evolution of the infected and susceptible vertices over the subsequent iterations of the Edge-Revelation algorithm, we make the following elementary observation. For any sequence $a_{n}=o(n)$, on the event $\left\{A_{t}^{\tau-1} \leq a_{n}\right\}$, we have, using Taylor expansion for the binomial probabilities:

$$
\begin{aligned}
\mathbb{P}\left[\operatorname{Bin}\left(A_{t}^{\tau-1}, c / n\right) \geq 1 \mid \mathcal{F}_{\tau-1}\right] & =1-\left(1-\frac{c}{n}\right)^{A_{t}^{\tau-1}}=1-\left(1-A_{t}^{\tau-1} \frac{c}{n}+O\left(\frac{\left(c A_{t}^{\tau-1}\right)^{2}}{n^{2}}\right)\right) \\
& =(1+o(1)) A_{t}^{\tau-1} \frac{c}{n} .
\end{aligned}
$$


Similarly, on the event $\left\{A_{t}^{\tau-1} \leq a_{n}\right\}$,

$$
\begin{aligned}
& \mathbb{P}\left[\operatorname{Bin}\left(A_{t}^{\tau-1}, c / n\right) \geq 2 \mid \mathcal{F}_{\tau-1}\right] \\
& =1-\left(1-\frac{c}{n}\right)^{A_{t}^{\tau-1}}-\frac{c}{n} A_{t}^{\tau-1}\left(1-\frac{c}{n}\right)^{A_{t}^{\tau-1}-1} \\
& =1-\left(1-A_{t}^{\tau-1} \frac{c}{n}+\frac{c^{2}}{2 n^{2}}\left(A_{t}^{\tau-1}\right)\left(A_{t}^{\tau-1}-1\right)+o\left(\frac{\left(c A_{t}^{\tau-1}\right)^{2}}{n^{2}}\right)\right) \\
& -\frac{c}{n} A_{t}^{\tau-1}\left(1-\left(A_{t}^{\tau-1}-1\right) \frac{c}{n}+O\left(\frac{\left(c A_{t}^{\tau-1}\right)^{2}}{n^{2}}\right)\right) \\
& =(1+o(1))\left(\frac{\left(c A_{t}^{\tau-1}\right)^{2}}{2 n^{2}}\right) \text {. }
\end{aligned}
$$

The algorithm terminates at the first step $\tau \geq 1$, such that $\overline{\mathcal{S}}_{t}^{\tau} \cup \overline{\mathcal{H}}_{t}^{\tau}=\emptyset$. Upon termination at $\tau$, we have that $\mathcal{I}_{t}^{\tau}=\mathcal{I}_{t}$. We show that when $t=o\left(n^{2 / 3}\right)$, then with high probability this algorithm terminates in $\tau=2$; moreover, $I_{t}=I_{t}^{\tau=2}=o\left(n^{2 / 3}\right)$ w.h.p.

We analyze the rounds of the algorithm sequentially. We start with $\tau=0$, and note that $I_{t}^{0}=B_{t}=2 t=T=o\left(n^{2 / 3}\right)$. For $\tau=1$, we get:

$$
\begin{aligned}
\hat{H}_{t}^{1} & =\operatorname{Bin}\left(H_{t}^{0}, \mathbb{P}\left[\operatorname{Bin}\left(A_{t}^{0}, c / n\right)=1\right]\right), \\
\bar{H}_{t}^{1} & =\operatorname{Bin}\left(2, \mathbb{P}\left[\operatorname{Bin}\left(A_{t}^{0}, c / n\right)=1\right]\right)+\operatorname{Bin}\left(H_{t}^{0}, \mathbb{P}\left[\operatorname{Bin}\left(A_{t}^{0}, c / n\right) \geq 2\right]\right), \\
\bar{S}_{t}^{1} & =\operatorname{Bin}\left(S_{t}^{0}, \mathbb{P}\left[\operatorname{Bin}\left(A_{t}^{0}, c / n\right) \geq 1\right]\right)=0 .
\end{aligned}
$$

Direct computation yields $\mathbb{E}\left[\hat{H}_{t}^{1}\right]=o\left(n^{2 / 3}\right)$ and $\mathbb{E}\left[\bar{H}_{t}^{1}\right]=o\left(n^{1 / 3}\right)$. Using Markov inequality, this immediately implies that $\hat{H}_{t}^{1}=o\left(n^{2 / 3}\right)$ and $\bar{H}_{t}^{1}=o\left(n^{1 / 3}\right)$. This, in turn implies that

$$
\begin{aligned}
& S_{t}^{1}=S_{t}^{0}-\bar{S}_{t}^{1}+\hat{H}_{t}^{1}=T=o\left(n^{2 / 3}\right), H_{t}^{1}=H_{t}^{0}-\bar{H}_{t}^{1}-\hat{H}_{t}^{1}=\Theta(n), \\
& A_{t}^{1}=\bar{H}_{t}^{1}=o\left(n^{1 / 3}\right), I_{t}^{1}=I_{t}^{0}+A_{t}^{1}=o\left(n^{2 / 3}\right) .
\end{aligned}
$$

We next analyze the next round of the Edge Revelation algorithm. For $\tau=2$, we have

$$
\begin{aligned}
\hat{H}_{t}^{2} & =\operatorname{Bin}\left(H_{t}^{1}, \mathbb{P}\left[\operatorname{Bin}\left(A_{t}^{1}, c / n\right)=1\right]\right), \\
\bar{H}_{t}^{2} & =\operatorname{Bin}\left(H_{t}^{1}, \mathbb{P}\left[\operatorname{Bin}\left(A_{t}^{1}, c / n\right) \geq 2\right]\right), \\
\bar{S}_{t}^{2} & =\operatorname{Bin}\left(S_{t}^{1}, \mathbb{P}\left[\operatorname{Bin}\left(A_{t}^{1}, c / n\right) \geq 1\right]\right) .
\end{aligned}
$$

For any $\varepsilon>0$, we have, for $\delta_{1}>0$

$$
\mathbb{P}\left[\hat{H}_{t}^{2}>\varepsilon n^{1 / 3}\right] \leq \mathbb{P}\left[\hat{H}_{t}^{2}>\varepsilon n^{1 / 3}, A_{t}^{1}<\delta_{1} n^{1 / 3}\right]+o(1) \leq \frac{\delta_{1} c}{\varepsilon}+o(1),
$$

where the last inequality follows using Markov inequality, conditioned on $\mathcal{F}_{1}$. We note that as $\delta_{1}>0$ is arbitrary, $\hat{H}_{t}^{2}=o\left(n^{1 / 3}\right)$. A similar analysis reveals that $\bar{H}_{t}^{2}=o(1)$ and $\bar{S}_{t}^{2}=o(1)$. Armed with these observations, we immediately conclude that

$$
\begin{aligned}
& S_{t}^{2} \leq 4 A_{t}^{1}+S_{t}^{1}-\bar{S}_{t}^{2}+\hat{H}_{t}^{2}=o\left(n^{2 / 3}\right), H_{t}^{2}=H_{t}^{1}-\bar{H}_{t}^{1}-\hat{H}_{t}^{1}=\Theta(n), \\
& A_{t}^{2}=\bar{S}_{t}^{2}+\bar{H}_{t}^{2}=o(1), I_{t}^{2}=I_{t}^{1}+A_{t}^{1}=o\left(n^{2 / 3}\right) .
\end{aligned}
$$

The upper-bound in [1] is due to the $4 A_{t}^{1}$ term which accounts for the four neighbors of an additionally infected node (outside of $\mathcal{B}_{t}$ ) on the $\mathcal{C}_{2}$. The validity of this recursion hinges crucially on the observation that with high probability, two nodes that are infected at round 2 (belonging to $\mathcal{A}_{t}^{2}$ ) are at least distance two apart on $\mathcal{C}_{2}$. We state this assertion formally in the lemma below, and complete the proof assuming this lemma. We defer its proof to the end of the section.

Lemma S2.2. With high probability as $n \rightarrow \infty$, no two infected vertices outside $\mathcal{B}_{t}$ are neighbors on $\mathcal{C}_{2}$.

Note that after $\tau=2$ steps the algorithm terminates with high probability, as there are no additional infected nodes identified. This completes the proof. 
Finally, we turn to the proof of Lemma S2.2.

Proof of Lemma S2.2: Note that two neighboring nodes are infected in the first two rounds of the Edge Revelation algorithm provided there exist two vertices at distance at most two on $\mathcal{C}_{2}$ such that at least one is infected and the other node becomes susceptible. There are $\Theta(n)$ such pairs, and the probability of such an event is $\mathbb{P}\left(\operatorname{Bin}\left(A_{t}^{1}, c / n\right) \geq 1\right) \mathbb{P}\left(\operatorname{Bin}\left(A_{t}^{1}, c / n\right) \geq 2\right)$. The required result follows upon computing the expected number of such neighboring pairs on $\mathcal{C}_{2}$.

\section{B. Spreading time Upper-Bound for Complex Contagion.}

Theorem S2.3. With high probability, the entire graph will be infected in time $n^{2 / 3}(\log \log n)^{2}(1+o(1))$.

Proof of Theorem S2.3. We devise an algorithm to lower bound the initial growth of the set of infected nodes. Let us consider a sequence $a_{n} \rightarrow \infty$ as $n \rightarrow \infty$, to be specified later. Next, we divide the cycle into consecutive intervals of length $L=n^{2 / 3} a_{n}(\log \log n)^{2}$. Call an interval active if two neighboring nodes (an adjacent pair) within that interval are infected. Initially, declare the interval containing the original seed nodes as an active interval. Starting with the initial seed nodes, after $n^{2 / 3} a_{n}$ steps the length of the original interval grows to $2 n^{2 / 3} a_{n}$. In general, after $n^{2 / 3} a_{n}$ time steps the length of infected segments in each active interval will be at least $n^{2 / 3} a_{n}$ due to the deterministic growth along the cycle. By revealing the edges of $\mathcal{G}_{n, c / n}$ that are incident to the newly infected nodes at the end of each $n^{2 / 3} a_{n}$ epoch, we can identify new active intervals that will, in turn, grow and activate other intervals. Formally, we consider the following algorithm to undercount the number of infected nodes. Denote by $\mathcal{X}_{\tau}$ the set of active intervals after $\tau$ epochs of length $n^{2 / 3} a_{n}$ each, and let $X_{\tau}=\left|\mathcal{X}_{\tau}\right|$ be the number of such intervals.

\section{Algorithm: Interval-Growth}

- Initialize:

- Let $N=n / L=n^{1 / 3} /\left(a_{n}(\log \log n)^{2}\right)$. Divide the cycle into $N$ intervals of length $L$ each, and label them by $[N]$; let the initial seed nodes be contained in the interval that is labeled one: $\mathcal{X}_{0}=\{1\}$.

- For $\tau \geq 1$, given $\mathcal{X}_{\tau-1}$ :

- Infect $n^{2 / 3} a_{n}$ new nodes along the cycle in each of the active intervals. Add edges independently with probability $c / n$, connecting the newly infected nodes to the healthy nodes.

- Update the set of active intervals by adding all intervals that have two adjacent infected nodes due to the edge exposure operation in the previous step: $\mathcal{X}_{\tau} \leftarrow \mathcal{X}_{\tau-1}$.

The following lemma derives a lower bound on the number of active intervals after $O(\log \log n)$ steps.

Lemma S2.4 (Initial Growth of the intervals). For any $C>0$, there exists a sequence $t_{n}:=t_{n}(C)=o(\log \log n)$ such that for

$$
\tau_{n}=\frac{1}{\log 3}\left(\log \log n+t_{n}\right),
$$

with high probability as $n \rightarrow \infty, X_{\tau_{n}} \geq C n^{1 / 9}(\log n)^{1 / 3}$.

An extra $n^{2 / 3}$ epoch beyond $\tau_{n}$ ensures that each activated interval has at least $n^{2 / 3}$ infected nodes. The next Lemma shows that this is enough to guarantee that the whole graph will be infected in $\left(\tau_{n} a_{n}+2\right) n^{2 / 3}+2$ time.

Lemma S2.5 (End Regime). Let $T_{n}$ be the first time that 2-complex contagion infects $C n^{7 / 9}(\log n)^{1 / 3}$ nodes, where $C>2 / c$ is a universal constant. Then with high probability, all nodes are infected by time $T_{n}+n^{2 / 3}+2$.

This concludes the proof.

It remains to prove Lemma S2.4 and Lemma S2.5. We first turn to the proof of Lemma S2.4.

Proof of Lemma S2.4. Let us denote the natural filtration associated with the growth process outlined in the IntervalGrowth algorithm by $\left\{\mathcal{F}_{\tau}: \tau \geq 1\right\}$. Note that at the end of $\tau-1$ steps, we have $X_{\tau-1}$ infected intervals, and thus $N-X_{\tau-1}$ non-active intervals. Each non-active interval of length $L$ has at least $\frac{1}{2} L$ mutually disjoint adjacent pairs, 
and the interval is activated if any one of these adjacent pairs is infected in the next step of the Interval-Growth algorithm introduced above. The independence of the added edges naturally implies that given $\mathcal{F}_{\tau-1}$, we have:

$$
X_{\tau}=X_{\tau-1}+\operatorname{Bin}\left(N-X_{\tau-1}, p_{\tau}\right)
$$

where $p_{\tau}$ is the conditional probability that an interval is activated by the edge exposure operation in the IntervalGrowth algorithm. Given $\mathcal{F}_{\tau-1}$ we can lower bound $p_{\tau}$ as follows. Each non-active interval has at least $\frac{1}{2} L$ disjoint adjacent pairs of neighboring nodes, and thus if the edge exposure infects a node and creates at least one infected node for its neighbor, the interval is activated due to complex contagion along the cycle. Thus we have,

$$
p_{\tau} \geq \mathbb{P}\left[\operatorname{Bin}\left(\frac{1}{2} L, \mathbb{P}\left[\operatorname{Bin}\left(X_{\tau-1} n^{2 / 3} a_{n}, \frac{c}{n}\right) \geq 2\right] \mathbb{P}\left[\operatorname{Bin}\left(X_{\tau-1} n^{2 / 3} a_{n}, \frac{c}{n}\right) \geq 1\right]\right) \geq 1\right] .
$$

Set $\delta_{n} \rightarrow 0$ as $n \rightarrow \infty$. Consider a choice of $\tau_{n}$ satisfying the conditions of Lemma S2.4. On the event that $X_{\tau_{n}-1}>\varepsilon N$ for some $\varepsilon>0$, the claim holds automatically. Thus we have, on the event $\left\{X_{\tau-1} \leq N \delta_{n}\right\}$

$$
\mathbb{E}\left[X_{\tau}-X_{\tau-1}\right] \geq \frac{1}{4} X_{\tau-1}^{3}\left(a_{n} c\right)^{3} .
$$

Using Bernstein's inequality (lower tail bound), we have, for some universal constant $c_{0}>0$ with probability at least $1-2 \exp \left(-c_{0} a_{n}^{3}\right)$

$$
X_{\tau} \geq X_{\tau-1}^{3}\left(\frac{c a_{n}}{2}\right)^{3}
$$

Let us choose $\tau_{n}=\frac{\log \log n+t_{n}}{\log 3}$ for some sequence $t_{n}=t_{n}(C)=o(\log \log n)$. Then using union bound, the probability that we do not have the desired growth in the number of infected intervals in at least one of the rounds may be upper bounded by $\tau_{n} \exp \left(-c_{0} a_{n}^{3}\right)$. We note that if we choose $a_{n}=\log \log n$, the probability of this bad event is $o(1)$. Moreover, on the good event, we have, $X_{\tau_{n}} \geq\left(\frac{c a_{n}}{2}\right)^{3^{\tau_{n}}}$. With $\tau_{n}=\left(\log \log n+t_{n}\right) / \log 3$, we can choose $t_{n}$ appropriately such that $X_{\tau_{n}} \geq C n^{1 / 9}(\log n)^{1 / 3}$. Finally, note that in $\Theta(\log \log n)$ rounds, we grow to length $n^{2 / 3} a_{n} \log \log n$ on the first interval, which is $o(L)$, thus indicating that the growth in infected vertices is sustained throughout the first $\tau$ rounds. This establishes the desired lower bound on the number of activated intervals in the first $\tau$ rounds.

Finally, we prove Lemma S2.5.

Proof of Lemma S2.5. Fix any node $x$ in the graph, and consider the interval $\mathcal{L}_{x}$ along the $\mathcal{C}_{2}$ cycle with center at $x$ and of length $n^{2 / 3}$. We say that the interval $\mathcal{L}_{x}$ contains a susceptible pair if it contains two neighboring vertices on $\mathcal{C}_{2}$, at least one of which is infected, and the other (possibly uninfected) has at least one additional infected neighbor. We note that if an interval contains a susceptible pair at time $T_{n}, x$ will be infected by time $T_{n}+2+n^{2 / 3}$ due to complex contagion along the cycle. Thus without loss of generality, consider $x$ such that $\mathcal{L}_{x}$ does not contain a susceptible pair at time $T_{n}$. Let $A_{x}$ denote the event that $\mathcal{L}_{x}$ contains a susceptible pair at time $T_{n}+1$. For any fixed $x, \mathcal{L}_{x}$ has at least $\frac{1}{2} n^{2 / 3}$ disjoint pairs of adjacent vertices. Each such pair is susceptible if at least one node is infected and the other has an infected neighbor. Denoting the number of infected vertices at $T_{n}$ as $I_{T_{n}}$, we observe that a fixed pair becomes suscetible by time $T_{n}+1$ with probability at least $\mathbb{P}\left[\operatorname{Bin}\left(I_{T_{n}}, \frac{c}{n}\right) \geq 2\right] \mathbb{P}\left[\operatorname{Bin}\left(I_{T_{n}}, \frac{c}{n}\right) \geq 1\right]$. The probability of the complement is thus at most $\left(1-\mathbb{P}\left[\operatorname{Bin}\left(I_{T_{n}}, \frac{c}{n}\right) \geq 2\right] \mathbb{P}\left[\operatorname{Bin}\left(I_{T_{n}}, \frac{c}{n}\right) \geq 1\right]\right)$. Using independence of the adjacent pairs in $\mathcal{L}_{x}$, we have,

$$
\mathbb{P}\left(A_{x}^{C}\right) \leq\left(1-\mathbb{P}\left[\operatorname{Bin}\left(C n^{7 / 9}(\log n)^{1 / 3}, \frac{c}{n}\right) \geq 2\right] \mathbb{P}\left[\operatorname{Bin}\left(C n^{7 / 9}(\log n)^{1 / 3}, \frac{c}{n}\right) \geq 1\right]\right)^{\frac{1}{2} n^{2 / 3}}=o\left(\frac{1}{n}\right),
$$

for $C>1$ satisfying $C c>2$. Finally, a union bound over the vertices $x$ with no adjacent infected pair in $\mathcal{L}_{x}$ immediately implies that with high probability, every vertex has an infected adjacent pair within distance $n^{2 / 3}$ by time $T_{n}+1$. Thus we can guarantee that all vertices will be infected by time $T_{n}+n^{2 / 3}+2$, establishing the desired result. 


\section{S3. Complex contagion on $\mathcal{C}_{1}$ union random graph with simple adoptions along $\mathcal{C}_{1}$ (Theorem 2)}

In this section, we consider a model where in addition to complex contagion we have simple contagions along the $\mathcal{C}_{1}$ edges, i.e. contagion can spread with probability $q_{n}$ along $\mathcal{C}_{1}$. In this model, we compare the diffusion speed between $\mathcal{C}_{1} \cup \mathcal{G}_{n, 2 / n}$ and $\mathcal{C}_{2}$. We conclude that for large enough $q_{n}$, the contagion spreads faster in $\mathcal{C}_{1} \cup \mathcal{G}_{n, 2 / n}$. Hence, when there is a high enough (but vanishing with increasing $n$ ) probability of simple adoptions along the cycle-edges the rewiring of even the $\mathcal{C}_{2}$ edges will speed up the spread of complex contagions.

A. Lower-bound on the spreading time of contagion with simple adoptions. Throughout the subsequent discussion, we denote by $I_{t}$ the number of infected nodes by time $t$.

Theorem S3.1 (Upper-bounding the number of infected nodes until $t=o\left(\sqrt{n} / q_{n}\right)$ ). Fix $t=o\left(\sqrt{n} / q_{n}\right)$. Then with high probability as $n \rightarrow \infty, I_{t}=o(\sqrt{n})$.

Proof of Theorem S3.1. Let $\mathcal{B}_{t}$ be the largest interval on $\mathcal{C}_{1}$ containing the initial seed nodes and the neighboring nodes infected by simple contagion along the cycle in time $t$. Denoting the size of this set by $B_{t}$, note that $B_{t}$ is increasing in $t, B_{0}=2$ and $B_{t} \rightarrow n$ with probability one as $t \rightarrow \infty$. To complete the proof, we will use the following lemmas.

Lemma S3.2 (Duration of the Initial Simple Contagion Phase). For $t q_{n}=o(\sqrt{n}), B_{t}=o(\sqrt{n})$.

Lemma S3.3 (Initial Simple Contagion Phase). Fix $t q_{n}=o(\sqrt{n})$. With high probability as $n \rightarrow \infty$, none of the nodes outside $\mathcal{B}_{t}$ are infected.

The proof of Theorem S3.1 is complete given these lemmas. We prove these assertions in the rest of this section.

First we turn to the proof of Lemma S3.2. We will utilize the following lower tail bound on negative binomial random variables.

Lemma S3.4 (Lower Tail Bounds for Geometric Variables). Let $\mathbf{g}_{i}, i=1, \ldots, n$ be a sequence of i.i.d. geometric variables with mean $1 / \hat{q}_{n}, \hat{x}=\hat{q}_{n}$ t and consider $\mathbf{G}(\hat{x}):=\sum_{i=1}^{\lfloor\hat{x}\rfloor} \mathbf{g}_{i}$. If $t \hat{q}_{n} \rightarrow \infty$ as $n \rightarrow \infty$, then $\mathbf{G}(\hat{x})>t / 2$ with high probability.

Proof of Lemma S3.4. Let $Y_{1}, Y_{2}, \ldots$ be a sequence of i.i.d. Bernoulli variables with success probability $\hat{q}_{n}$. Then $\mathbf{G}(\hat{x})$ has the same (negative binomial) distribution as the smallest $j$ such that exactly $\lfloor\hat{x}\rfloor$ of $Y_{1}, \ldots, Y_{j}$ are one (see e.g. (6)). In particular, $\mathbf{G}(\hat{x})<t / 2$ if, and only if, $\sum_{i=1}^{\lfloor t / 2\rfloor} Y_{i}>\hat{x}=\hat{q}_{n} t$. We can bound the probability of the latter event by a simple application of Markov inequality for the sum of i.i.d. Bernoulli variables. We have,

$$
\mathbb{P}\left\{\sum_{i=1}^{\lfloor t / 2\rfloor} Y_{i}>\hat{q}_{n} t\right\} \leq \frac{\mathbb{E}\left\{\left(\sum_{i=1}^{\lfloor t / 2\rfloor} Y_{i}\right)^{2}\right\}}{\hat{q}_{n}^{2} t^{2}}=\frac{(t / 2) \hat{q}_{n}}{\hat{q}_{n}^{2} t^{2}}=\frac{1}{2 t \hat{q}_{n}}=o(1) .
$$

Hence, $\mathbb{P}\{\mathbf{G}(\hat{x}) \geq t / 2\} \rightarrow 1$ as $n \rightarrow \infty$, completing the proof.

Armed with Lemma S3.4, we can now establish Lemma S3.2.

Proof of Lemma S3.2. Let $t=o\left(\sqrt{n} / q_{n}\right)$ and consider the spread of simple contagion along the $\mathcal{C}_{1}$ edges up to time $t$. Starting from two adjacent seeds, the time for the simple contagion to spread to either neighboring node is a geometric random variable with success probability $q_{n}$ and mean $1 / q_{n}$. In particular, the time that it takes until the first of the two nodes (at either sides of the two adjacent initial seeds) gets infected is the minimum of two i.i.d. geometric mean $1 / q_{n}$ variables, which is another geometric variable with success probability $\hat{q}_{n}=1-\left(1-q_{n}\right)^{2}=(2+o(1)) q_{n}$. To upper-bound the number of simple infections up to time $t=o\left(\sqrt{n} / q_{n}\right)$ we can consider a sped up infection process whereby whenever the first of the two nodes at either side of the infected interval is infected we force the second one to be infected as well. Denote the infected interval that results form this sped-up simple infection process by $\hat{\mathcal{B}}_{t}$, and set $\hat{B}_{t}=\left|\hat{\mathcal{B}}_{t}\right|$. First we show that $\hat{B}_{t}=o(\sqrt{n})$ for $t=o\left(\sqrt{n} / q_{n}\right)$. To this end, let $\mathbf{g}_{i}, i=1, \ldots, n$ be a sequence of i.i.d. geometric variables with mean $1 / \hat{q}_{n}$, and $\mathbf{x}^{\star}:=\sup \left\{x: \sum_{i=1}^{x} \mathbf{g}_{i}<t\right\}$. The random variable $\mathbf{x}^{\star}$ measures the growth of $\hat{\mathcal{B}}_{t}$ at both ends until time $t$; hence, $\hat{B}_{t}=2+2 \mathbf{x}^{\star}$. Let $\hat{x}=2 \hat{q}_{n} t$ and consider $\mathbf{G}(\hat{x}):=\sum_{i=1}^{\lfloor\hat{x}\rfloor} \mathbf{g}_{i}$. Lemma S3.4 implies that $\mathbf{G}(\hat{x})>t$ with high probability whenever $t q_{n} \rightarrow \infty$ as $n \rightarrow \infty$. Thus $\hat{B}_{t}=2+2 \mathbf{x}^{\star}<2+2 \hat{x}=2+4 t \hat{q}_{n}=o(\sqrt{n})$ with high probability. Finally, we show that with high probability as $n \rightarrow \infty, \mathcal{B}_{t} \subset \hat{\mathcal{B}}_{t}$. Note that $\mathcal{B}_{t} \not \subset \hat{\mathcal{B}}_{t}$ only if 
there is at least one vertex which is infected by complex contagion up to time $t$. We note that occurs with probability $o(1)$ by Lemma S3.3. This establishes that $\mathcal{B}_{t} \subset \hat{\mathcal{B}}_{t}$ with high probability. The above completes the proof for the case $t \hat{q}_{n} \rightarrow \infty$. The case $t \hat{q}_{n}=O(1)$ follows from the monotonicity of $B_{t}$.

Finally we establish Lemma S3.3.

Proof of Lemma S3.3. Fix $t=o\left(\sqrt{n} / q_{n}\right)$ and note that Lemma S3.2 implies that there exists a sequence $a_{n}=o(\sqrt{n})$ such that $\mathbb{P}\left(B_{t}<a_{n}\right)=1-o(1)$. Now, conditional on $\left\{B_{t}<a_{n}\right\}$, note that for contagion to spread to some node outside of $\mathcal{B}_{t}$, there has to be at least one node outside of $\mathcal{B}_{t}$ that has at least two long ties to the nodes inside $\mathcal{B}_{t}$. Therefore

$\mathbb{P}\left\{\right.$ Some node outside $\mathcal{B}_{t}$ is infected $\}$

$\leq \mathbb{P}\left\{\right.$ Some nodes outside $\mathcal{B}_{t}$ has two long-tie to the nodes inside $\}$

$$
\leq \mathbb{P}\left\{\operatorname{Bin}\left(n-B_{t}, \mathbb{P}\left[\operatorname{Bin}\left(B_{t}, 2 / n\right) \geq 2\right]\right) \geq 1\right\}=O\left(\left(n-B_{t}\right) \frac{4 B_{t}^{2}}{n^{2}}\right)=o(1) .
$$

Hence for $t=o\left(\sqrt{n} / q_{n}\right)$, with high probability all infected nodes belong to $\mathcal{B}_{t}$, and $\mathcal{I}_{t}=\mathcal{B}_{t}$. This completes the proof.

B. Upper-bound on the spreading time of contagion with simple adoptions. The next result derives an upper bound on the infection time of all nodes in the graph. Some aspects of the proof are similar to that of Theorem S2.3.

Theorem S3.5. With high probability, the entire graph will be infected in time $3 \frac{\sqrt{n}}{q_{n}}(\log \log n)^{2}(1+o(1))$.

Proof of Theorem S3.5. As in the proof of Theorem S2.3, we devise an algorithm to lower bound the initial growth of the set of infected nodes. Next, we divide the cycle into consecutive intervals of length $L=\sqrt{n}(\log \log n)^{3}$. Call an interval active if it contains an infected node. Initially, declare the interval containing the original seed nodes, an active interval. Starting with the initial seed nodes, after $2 \sqrt{n} \log \log n / q_{n}$ steps the length of the original interval grows to at least $\sqrt{n} \log \log n$ with high probability. In general, with high probability as $n \rightarrow \infty$, over the first $O(\log \log n)$ iterations of this algorithm, after each set of $2 \sqrt{n} \log \log n / q_{n}$ time steps, the length of infected segments in each active interval will grow at least $\sqrt{n} \log \log n$ due to the simple contagion along the cycle. By revealing the edges of $\mathcal{G}_{n, 2 / n}$ that are incident to the newly infected nodes at the end of each $2 \sqrt{n} \log \log n / q_{n}$ epoch, we can identify new active intervals that will, in turn, grow and activate other intervals. Formally, we consider the following algorithm to undercount the number of infected nodes. Denote by $X_{\tau}$ the number of active intervals after $\tau$ epochs of length $2 \sqrt{n} \log \log n / q_{n}$ each.

\section{Algorithm: Super-Exponential-Activation}

- Initialize:

- Let $N=\sqrt{n} /(\log \log n)^{3}$. Divide the cycle into $N$ intervals of length $L_{n}=\sqrt{n}(\log \log n)^{3}$ each, and label them by $[N]$; let the initial seed nodes be contained in the interval that is labeled one: $\mathcal{X}_{0}=\{1\}$.

- For $\tau \geq 1$, given $\mathcal{X}_{\tau-1}$ :

- Wait for an epoch of length $2 \sqrt{n} \log \log n / q_{n}$ and track the growth of infected nodes along the cycle by simple contagion during this epoch.

- Add edges independently with probability $2 / n$ connecting the new infected nodes to the healthy nodes.

- Update the set of active intervals by adding all intervals that have new infected nodes in them: $\mathcal{X}_{\tau} \leftarrow \mathcal{X}_{\tau-1}$.

The first lemma derives a lower bound on the number of active intervals after $O(\log \log n)$ steps.

Lemma S3.6 (Initial Growth of the intervals). For any $C>0$, there exists a sequence $t_{n}:=t_{n}(C)=o(\log \log n)$ such that for

$$
\tau_{n}=\frac{1}{\log 2}\left(\log \log n+t_{n}\right),
$$

with high probability as $n \rightarrow \infty, X_{\tau_{n}} \geq C n^{1 / 4}(\log n)^{1 / 2}$. 
Finally, the next result states that with high probability, the contagion process spreads to the whole graph in time $\left(\tau_{n}\right)\left(2 \sqrt{n} \log \log n / q_{n}\right)+1+2 \sqrt{n} / q_{n}$.

Lemma S3.7 (End Regime). Let $T_{n}$ be the first time that simple contagion infects at least $C n^{3 / 4} \sqrt{\log n}$ nodes, where $C>1$ is a universal constant. Then with high probability, all nodes are infected by time $T_{n}+1+2 \sqrt{n} / q_{n}$.

The proof follows upon combining Lemma S3.6 and Lemma S3.7, and noting that $1 / \log 2<3 / 2$.

We turn to the proof of Lemma S3.6 and Lemma S3.7. We first establish that for the first $O(\log \log n)$ rounds, at least $\sqrt{n} \log \log n$ new nodes are infected over each $2 \sqrt{n} \log \log n / q_{n}$ epoch. To this end, we require the following lemma.

Lemma S3.8. Fix $\tau_{n}=\frac{1}{\log 2} \log \log n(1+o(1))$. With high probability as $n \rightarrow \infty$, every activated interval gains at least $\sqrt{n} \log \log n$ new infected nodes over each of the first $\tau_{n}$ epochs of length $2 \sqrt{n} \log \log n / q_{n}$.

Proof of Lemma S3.8. We begin with the observation that the waiting time for an activated interval to gain at least $\sqrt{n} \log \log n$ new vertices via simple contagion is a sum of $\sqrt{n} \log \log n$ i.i.d. Geometric random variables, each with mean $1 / q_{n}$. Let $\left\{g_{1}, g_{2}, \cdots\right\}$ be a sequence of i.i.d. Geometric random variables with mean $1 / q_{n}$. Thus during an epoch of length $2 \sqrt{n} \log \log n / q_{n}$, an activated interval fails to gain at least $\sqrt{n} \log \log n$ new infected nodes via simple contagion along the circle if $\sum_{i=1}^{\sqrt{n} \log \log n} g_{i}>2 \sqrt{n} \log \log n / q_{n}$. Standard upper tail bounds on sums of geometric random variables $(6$, Theorem 1.14$)$ directly implies that

$$
\mathbb{P}\left[\sum_{i=1}^{\sqrt{n} \log \log n} \mathbf{g}_{i}>2 \frac{\sqrt{n} \log \log n}{q_{n}}\right] \leq \exp \left(-\frac{\sqrt{n} \log \log n}{4}\right) .
$$

Note that at each epoch, there are at most $N=\sqrt{n} /(\log \log n)^{3}<\sqrt{n}$ active intervals. Thus a direct union bound argument over $O(\log \log n)$ rounds of the algorithm establishes that with high probability, each activated interval gains the desired number of infected vertices in at each epoch. It is implicit in the calculation above that the infection of new vertices is sustained as long as an active interval is not exhausted. To show this sustained growth, note that over a time epoch of length $2 \sqrt{n} \log \log n / q_{n}$, the probability an active interval gains more than $M \sqrt{n} \log \log n$ new infected vertices, for some $M>1$, can be upper-bounded as follows:

$$
\mathbb{P}\left[\sum_{i=1}^{M \sqrt{n} \log \log n} g_{i}<2 \frac{\sqrt{n} \log \log n}{q_{n}}\right] \leq \exp \left(-\frac{1}{2} \log M \sqrt{n} \log \log n\right),
$$

where the last inequality follows using (7, Theorem 3.1). Thus for $M>1$ sufficiently large, by union bound over the $N=\sqrt{n} /(\log \log n)^{3}$ intervals in $\tau_{n}=\frac{1}{\log 2} \log \log n(1+o(1))$ rounds, we can guarantee that at each epoch, every active interval gains at most $M \sqrt{n} \log \log n$ many new nodes by simple infection. Hence, no active interval is exhausted in the first $\tau_{n}=\frac{1}{\log 2} \log \log n(1+o(1))$ rounds with high probability as $n \rightarrow \infty\left(\right.$ since $\left.L_{n}=\sqrt{n}(\log \log n)^{3}\right)$. This completes the proof.

Given Lemma S3.8, we can now prove Lemma S3.6.

Proof of Lemma S3.6. Let us denote the natural filtration associated with the Super-Exponential-Activation growth process as $\left\{\mathcal{F}_{\tau}: \tau \geq 1\right\}$. Note that at the end of $\tau-1$ steps, we have $X_{\tau-1}$ infected intervals, and thus $N-X_{\tau-1}$ non-active intervals. Each non-active interval is activated if at least one node has two infected neighbors in the Super-Exponential-Activation algorithm described above. The independence of the edges added naturally implies that given $\mathcal{F}_{\tau-1}$, we have,

$$
X_{\tau}=X_{\tau-1}+\operatorname{Bin}\left(N-X_{\tau-1}, p_{\tau}\right),
$$

where $p_{\tau}$ is the conditional probability that an interval is activated by the edge exposure operation in the SuperExponential-Growth algorithm. Let $\mathcal{E}$ denote the good event that each active interval has gained at least $\sqrt{n} \log \log n$ new infected vertices during each epoch of length $2 \sqrt{n} \log \log n / q_{n}$ up to the $\tau^{\text {th }}$ round. Observe that Lemma S3.8 implies that $\mathbb{P}(\mathcal{E})=1-o(1)$. Now, given $\mathcal{F}_{\tau-1}$, we can lower bound $p_{\tau}$ on the event $\mathcal{E}$ as follows.

$$
p_{\tau} \geq \mathbb{P}\left[\operatorname{Bin}\left(L, \mathbb{P}\left[\operatorname{Bin}\left(X_{\tau-1} \sqrt{n} \log \log n, \frac{2}{n}\right) \geq 2\right]\right) \geq 1\right] .
$$


On the event that $X_{\tau-1}>\varepsilon N$ for some $\varepsilon>0$, the claim is automatically satisfied. Thus we set $\delta_{n} \rightarrow 0$ as $n \rightarrow \infty$, and note that on the event $\left\{X_{\tau-1} \leq N \delta_{n}\right\} \cap \mathcal{E}$

$$
\mathbb{E}\left[X_{\tau}-X_{\tau-1}\right] \geq X_{\tau-1}^{2}(\log \log n)^{2} .
$$

Using Bernstein's inequality, we have, for some universal constant $c_{0}>0$ with probability at least $1-2 \exp \left(-c_{0}(\log \log n)^{2}\right)$

$$
X_{\tau} \geq X_{\tau-1}^{2}\left(\frac{\log \log n}{2}\right)^{2} .
$$

Let us choose $\tau_{n}=\frac{\log \log n+t_{n}}{\log 2}$ for some sequence $t_{n}=t_{n}(C)=o(\log \log n)$. Then using union bound, the probability that we do not have the desired growth in the number of infected intervals in at least one of the rounds may be upper bounded by $\tau_{n} \exp \left(-c_{0}(\log \log n)^{2}\right)$. We note that the probability of this bad event is $o(1)$. Moreover, on the good event, we have, $X_{\tau_{n}} \geq\left(\frac{\log \log n}{2}\right)^{2^{\tau}}$. We choose $t_{n}$ appropriately such that $X_{\tau_{n}} \geq C n^{1 / 4} \sqrt{\log n}$. This establishes the desired lower bound on the number of infected nodes in the first $\tau$ rounds.

Finally we establish Lemma S3.7.

Proof of Lemma S3.7. For a node $x$, let $\mathcal{L}_{x}$ denote the interval of length $\sqrt{n}$ on the cycle centered at $x$. We will show that with high probability, for each $x, \mathcal{L}_{x}$ contains at least one infected node by time $T_{n}+1$. This implies that with high probability, all vertices will be infected by time $T_{n}+1+2 \sqrt{n} / q_{n}$ due to simple contagion along the cycle. This last assertion follows using upper tail concentration for a sum of geometric random variables and union bound, exactly as described in the proof of Lemma S3.8. Thus we will omit this step in the subsequent argument.

By time $T_{n}$, we have at least $C n^{3 / 4} \sqrt{\log n}$ infected nodes. If any of these infected nodes fall in $\mathcal{L}_{x}$ then we are done. If not, then for any fixed $x, \mathcal{L}_{x}$ has $\sqrt{n}$ vertices, and the probability that each such vertex has less than two infected neighbors is at most $\mathbb{P}\left[\operatorname{Bin}\left(C n^{3 / 4} \sqrt{\log n}, \frac{2}{n}\right)<2\right]$. Using independence of the edges in $\mathcal{G}_{n, 2 / n}$, the probability that none of the vertices in $\mathcal{L}_{x}$ is infected by time $T_{n}+1$ is at most $\left(1-\mathbb{P}\left[\operatorname{Bin}\left(C n^{3 / 4} \sqrt{\log n}, \frac{2}{n}\right)=2\right]\right)^{\sqrt{n}}$. Let $A_{x}$ denote the event that none of the vertices in $\mathcal{L}_{x}$ are infected by time $T_{n}+1$. The analysis above and direct computation immediately implies

$$
\mathbb{P}\left(A_{x}\right) \leq n^{-C} .
$$

Finally, a union bound over all nodes on the cycle completes the proof, since $C>1$.

C. Simple adoption probabilities under logit and probit Activation functions. In Figure $4 \mathrm{~B}$ of the main text we present the simulation results for the spreading time of complex contagion over $\mathcal{C}_{1}$ union $\mathcal{G}_{n, 2 / n}$ for various probabilities of simple adoptions $q$. Our theoretical results in Theorem 2 suggests that having $q=\omega(1 / \sqrt{n})$ is enough to ensure a faster spread in $\mathcal{C}_{1} \cup \mathcal{G}_{n, 1 / n}$ compared to $\mathcal{C}_{2}$. Here we derive the corresponding conditions on the parameters of the logit and probit functions to ensure that $q=\omega(1 / \sqrt{n})$ under these classes of activation functions. Consider a probit activation function such that the probability of adoptions when the number of infected neighbors is $x$ is given by:

$$
\Phi_{\theta, \sigma_{n}}(x)=\int_{-\infty}^{\frac{x-\theta}{\sigma_{n}}} \frac{1}{\sqrt{2 \pi}} e^{-t^{2} / 2} d t
$$

We are interested in the asymptotic regime $\sigma_{n} \rightarrow 0$ as $n \rightarrow \infty$. We choose $\theta=1.5$ to ensure a high probability of adoption with two infected neighbors and a low (but non-zero) adoption probability with only one infected neighbor. There is no adoption when there are no infections in the agent's neighborhood. The probability of adoptions below threshold is given by:

$$
\hat{q}_{n}=\Phi_{1.5, \sigma_{n}}(1)=\int_{-\infty}^{-\frac{1}{2 \sigma_{n}}} \frac{1}{\sqrt{2 \pi}} e^{-t^{2} / 2} d t=\int_{\frac{1}{2 \sigma_{n}}}^{+\infty} \frac{1}{\sqrt{2 \pi}} e^{-t^{2} / 2} d t
$$

Using the Gaussian tail bounds for $x>0$, 


$$
\frac{1}{\sqrt{2 \pi}} \frac{x}{x^{2}+1} e^{-x^{2} / 2} \leq \int_{x}^{\infty} \frac{1}{\sqrt{2 \pi}} e^{-t^{2} / 2} d t \leq \int_{x}^{\infty} \frac{t}{x} \frac{1}{\sqrt{2 \pi}} e^{-t^{2} / 2} d t=\frac{e^{-x^{2} / 2}}{x \sqrt{2 \pi}}
$$

we get

$$
\frac{e^{-1 / 8 \sigma_{n}^{2}}}{\sqrt{2 \pi}}\left(\frac{2 \sigma_{n}}{1+4 \sigma_{n}^{2}}\right) \leq \hat{q}_{n} \leq 2 \sigma_{n} \frac{e^{-1 / 8 \sigma_{n}^{2}}}{\sqrt{2 \pi}}
$$

Hence,

$$
\hat{q}_{n}=\Theta\left(2 \sigma_{n} e^{-1 / 8 \sigma_{n}^{2}}\right)
$$

In particular, taking $\sigma_{n}=1 / \sqrt{8 \log n^{\alpha}}$ yields that

$$
\hat{q}_{n}=\Theta\left(\frac{1}{\sqrt{2 \log n^{\alpha}}} n^{-\alpha}\right)
$$

Hence, $\hat{q}_{n}=o\left(n^{-1 / 2}\right)$ for $\alpha \geq 1 / 2$ and $\hat{q}_{n}=\omega\left(n^{-1 / 2}\right)$ for $\alpha<1 / 2$.

We can repeat the same calculations when the activation functions are specified by a logistic function:

$$
\Psi_{\theta, \sigma_{n}}(x)=\frac{1}{1+e^{\left(1 / \sigma_{n}\right)(\theta-x)}} .
$$

The probability of adoptions below threshold for logistic activation functions is given by:

$$
\tilde{q}_{n}=\Psi_{1.5, \sigma_{n}}(1)=\frac{1}{1+e^{\left(1 / 2 \sigma_{n}\right)}}=\Theta\left(e^{-1 / 2 \sigma_{n}}\right)
$$

for $\sigma_{n} \rightarrow 0$ as $n \rightarrow \infty$. Choosing $\sigma_{n}=\frac{1}{2 \log \left(n^{\alpha}\right)}$ yields $\tilde{q}_{n}=\Theta\left(n^{\alpha}\right): \tilde{q}_{n}=o\left(n^{-1 / 2}\right)$ for $\alpha>1 / 2$ and $\tilde{q}_{n}=\omega\left(n^{-1 / 2}\right)$ for $\alpha<1 / 2$.

Note that $\Phi_{\theta, \sigma}(0)>0$ and $\Psi_{\theta, \sigma}(0)>0$; hence, the logit and probit activation functions allow for a small probability of "spontaneous" adoptions even when there are no infected neighboring nodes.

\section{S4. Complex contagion over $\mathcal{C}_{2}^{\eta}$ with simple adoptions along $\mathcal{C}_{1}$ (Theorem 3)}

Theorems S2.1 and S2.3 characterize the time needed for all nodes to be infected by 2-complex contagion on $\mathcal{C}_{2} \cup \mathcal{G}_{n, 2 / n}$. In comparison, pure complex 2-contagion on $\mathcal{C}_{4}$ requires $\Theta(n)$ time to infect all vertices, although the average degree is the same in the two cases. One could naturally envision obtaining $\mathcal{C}_{2} \cup \mathcal{G}_{n, 2 / n}$ by a "re-wiring" of the edges in $\mathcal{C}_{4}$, and thus from a network intervention viewpoint, the result strongly suggests the usefulness of adding long edges to speed up the complex contagion procedure. However, any further rewiring destroys the 2-core necessary for the spread of complex contagion, and thus will actually stall the infection procedure. Theorems S3.1 and S3.5 amend this picture, and conclude that with the additional presence of a small probability of adoption below threshold, the inclusion of long edges significantly speeds up the infection procedure even by replacing the $\mathcal{C}_{2}$ edges.

In this section, we adopt a more dynamical viewpoint, and interpolate between $\mathcal{C}_{2}$ and $\mathcal{C}_{1} \cup \mathcal{G}_{n, 2 / n}$. To facilitate the spread of the infection process on the whole graph, we add a small probability $q_{n}$ of adoption below threshold along $\mathcal{C}_{1}$. Surprisingly, our findings below indicate that initial rewiring slows down the infection process, whereas after a point, provided $q_{n}$ is large enough, the infection process spreads faster compared to that of complex contagion on $\mathcal{C}_{2}$.

Formally, we consider the following interpolation model. Consider two random graph processes $\left\{\mathcal{D}_{\eta}, \eta \geq 0\right\}$ and $\left\{\mathcal{G}_{\eta}, \eta \geq 0\right\}$ that are coupled through the common index $\eta \geq 0$. For any fixed $\eta>0$, the graph processes are distributed as follows.

1. Let $\left\{X_{i j}: 1 \leq i<j \leq n\right\}$ be i.i.d. Exponential random variables with rate $1 / n^{2}$. Construct $\mathcal{G}_{\eta}$ with vertex set $[n]$, and for any $i<j$, we add an edge connecting the two vertices if $\left\{X_{i j}<\eta\right\}$. Therefore, the random graph $\mathcal{G}_{\eta}$ is Erdős-Rényi with edge probability $\mathbb{P}\left\{X_{i j}<\eta\right\}=1-e^{-\eta / n^{2}}$. 
2. Associate with every edge $i, j$ in $\mathcal{C}_{2} \backslash \mathcal{C}_{1}$ an independent exponential variable $Y_{i j}$ with mean $2 n$. Retain edge $i, j$ in $D_{\eta} \subset \mathcal{C}_{2} \backslash C_{1}$ if $Y_{i, j}>\eta$. Thus the probability that the cycle edge $i, j$ is removed is $1-e^{-\eta / 2 n}$.

We will study the spread of contagion on the interpolated graph $\mathcal{C}_{2}^{\eta}:=\mathcal{C}_{1} \cup \mathcal{G}_{\eta} \cup \mathcal{D}_{\eta}$ in the regime $\eta=o(n)$. Note that for $\eta=o(n)$, the expected degree of a node in $\mathcal{C}_{2}^{\eta}$ is

$$
2+(n-3)\left(1-e^{-\eta / n^{2}}\right)+2 e^{-\eta / n}=2+(n-3)\left(\frac{\eta}{n^{2}}+o\left(\frac{\eta}{n^{2}}\right)\right)+2\left(1-\frac{\eta}{2 n}+o(\eta / n)\right)=4+o(\eta / n) .
$$

Hence, for $\eta=o(n)$ the expected average degree in $\mathcal{C}_{2}^{\eta}$ remain fixed and equal to four, which is the degree of nodes in $\mathcal{C}_{2}$. In this formulation, $\eta$ is asymptotically twice the expected number of edges that are rewired to construct $\mathcal{C}_{2}^{\eta}$ from $\mathcal{C}_{2}$.

For the subsequent analysis, we parametrize $\eta=n^{\delta}$ and study the infection spread over $\mathcal{C}_{2}^{\eta}$ in two regimes.

(i) For $0<\delta<1 / 2$ we show that the spread slows down with increasing $\eta$. In this regime, two long-ties are unlikely to land on the same node, and thus complex contagion along the long ties is blocked. On the other hand, the rewiring creates single edges along the cycle, which slows down the spread of contagion along the cycle (for a formal statement see Theorem S4.2 below).

(ii) For $1 / 2<\delta<1$ we show that contagion takes $O^{*}\left(n^{3 / 2} / \eta+\sqrt{n} / q_{n}\right)$ time to spread. For $\eta$ large enough, $\sqrt{n} / q_{n}$ is the dominant term that fixes the spreading speed. However, for $q_{n} \gg 1 / \sqrt{n}$ we can specify a range of $\eta$ for which increasing $\eta$ increases the speed, with complex contagion spreading through the long-ties. In particular, if $q_{n}=n^{-1 / 2+\epsilon}$, then for $1 / 2<\delta<1 / 2+\epsilon$, contagion spreads faster for larger values of $\eta$ (see Theorem S4.6 below).

A. Speed of Contagion for $0<\delta<1 / 2$. We establish a lower bound on the speed of contagion for $\delta \in\left(0, \frac{1}{2}\right)$ in this section. We need the following preliminary lemmas. For any graph $G$, let $\operatorname{deg}_{\min }(G)$ denote the minimum $\operatorname{degree}$ in $G$.

Lemma S4.1 (Unlikely occurrence of complex contagions). For $\eta=o(\sqrt{n}), \mathbb{P}\left(\operatorname{deg}_{\min }\left(\mathcal{G}_{\eta}\right) \geq 2\right)=o(1)$.

Proof of Lemma S4.1. Note that for any vertex $i \in[n]$, its degree in the induced subgraph is distributed as $\operatorname{Bin}(n, 1-$ $\left.e^{-\eta / n^{2}}\right)$. Thus the probability that a fixed vertex has degree at least two is

$$
\mathbb{P}\left(\operatorname{Bin}\left(n, 1-\exp \left(-\eta / n^{2}\right)\right) \geq 2\right)=O\left(\frac{\eta^{2}}{n^{2}}\right) .
$$

Thus the expected number of vertices with degree at least two in the subgraph induced by $G_{\eta}$ is $O\left(\eta^{2} / n\right)=o(1)$. This concludes the proof.

Armed with this lemma, we can now provide a lower bound on the time to contagion in this regime.

Theorem S4.2 (Lower-bound on the spreading time). With high probability as $n \rightarrow \infty$, the total spreading time is at least $\frac{n}{2}+\frac{\eta}{4 q_{n}}$.

Proof of Theorem S4.2. Fix $\delta \in\left(0, \frac{1}{2}\right)$. First, note that the number of edges deleted in $\mathcal{C}_{2} \backslash \mathcal{C}_{1}$ is distributed as $\operatorname{Bin}\left(n, 1-\exp \left(-\frac{\eta}{2 n}\right)\right)$. We denote this number as $M_{\eta}$. Next, observe that Lemma S4.1 implies that with high probability, none of the vertices may be infected by complex contagion, and the infection has to spread along the cycle. To pass each missing edge in $\mathcal{C}_{2} \backslash \mathcal{C}_{1}$, one incurs an independent $\operatorname{Geo}\left(q_{n}\right)$ waiting time. Setting $\tau$ as the time for contagion, we have,

$$
\mathbb{P}\left(\tau<\frac{n}{2}+\frac{\eta}{4 q_{n}}\right) \leq \mathbb{P}\left(\sum_{i=1}^{M_{\eta}} \tau_{i}-M_{\eta}<\frac{\eta}{4 q_{n}}\right) \leq \mathbb{P}\left(\sum_{i=1}^{M_{\eta}} \tau_{i}<\frac{\eta}{4 q_{n}}(1+o(1))\right)+o(1),
$$

where $\left\{\tau_{i}: i \geq 1\right\}$ are i.i.d. $\operatorname{Geo}\left(q_{n}\right)$ random variables and the second inequality follows since $\mathbb{E}\left[M_{\eta}\right]=\eta / 2=o\left(\eta / q_{n}\right)$. By direct computation

$$
\begin{aligned}
\mathbb{E}\left[\sum_{i=1}^{M_{\eta}} \tau_{i}\right]=\mathbb{E}\left[M_{\eta}\right] \mathbb{E}\left[\tau_{i}\right]=\frac{\eta}{2 q_{n}}(1+o(1)) . \\
\operatorname{Var}\left[\sum_{i=1}^{M_{\eta}} \tau_{i}\right]=\frac{1}{q_{n}^{2}} \operatorname{Var}\left(M_{\eta}\right)+\frac{1-q_{n}}{q_{n}^{2}} \mathbb{E}\left[M_{\eta}\right]=O\left(\frac{\eta}{q_{n}^{2}}\right) .
\end{aligned}
$$


Finally, using Chebychev's inequality, we have,

$$
\mathbb{P}\left(\sum_{i=1}^{M_{\eta}} \tau_{i}<\frac{\eta}{4 q_{n}}(1+o(1))\right) \leq 4 \frac{\operatorname{Var}\left[\sum_{i=1}^{M_{\eta}} \tau_{i}\right]}{\mathbb{E}^{2}\left[\sum_{i=1}^{M_{\eta}} \tau_{i}\right]}=O\left(\frac{1}{\eta}\right)=o(1) .
$$

This completes the proof.

B. Speed of Contagion for $\delta \in\left(\frac{1}{2}, 1\right)$. Let us fix $\delta \in\left(\frac{1}{2}, 1\right)$. Let us first concentrate on the spread of infection along the cycle via simple or complex contagion. We initialize the process by infecting two adjacent nodes. The infection spreads via complex two contagion until an edge of $\mathcal{C}_{2}$ is missing, when it pays a geometric waiting time. Thus to control how many vertices are infected over certain time periods, one needs a good control on the number of missing $\mathcal{C}_{2}$ edges in any sub-interval. The following lemma provides this necessary control.

Lemma S4.3. Consider an interval $\mathcal{I}$ of length $L$ on the cycle. Let $\mathcal{N}(\mathcal{I})$ denote the number of missing $\mathcal{C}_{2}$ edges on this segment. For $\lambda \in(0,1)$

$$
\mathbb{P}[|\mathcal{N}(\mathcal{I})-\mathbb{E}[\mathcal{N}(\mathcal{I})]|>\lambda \mathbb{E}[\mathcal{N}(\mathcal{I})]] \leq 2 \exp \left(-\frac{\lambda^{2}}{3} \mathbb{E}[\mathcal{N}(\mathcal{I})]\right)
$$

Proof of Lemma S4.3. We observe that for any fixed interval $\mathcal{I}$ of length $L$, the number of missing cycle edges $\mathcal{N}(\mathcal{I}) \sim \operatorname{Bin}(L, 1-\exp (-\eta / 2 n))$. The thesis follows by a direct application of Chernoff inequality for Binomial random variable.

Given Lemma S4.3, we can turn to deriving a lower bound on the spreading time for the infection on the cycle.

Theorem S4.4 (High probability lower-bound on the spreading time). Fix $t=o\left(\sqrt{n} / q_{n}+n^{3 / 2-\delta}\right)$. With high probability as $n \rightarrow \infty$, the number of infected vertices is $o\left(n^{3 / 2-\delta}\right)$.

Proof of Theorem S4.4. Fix $t=o\left(\sqrt{n} / q_{n}+n^{3 / 2-\delta}\right)$. For any $\varepsilon>0$, consider an interval of length $L=\varepsilon n^{3 / 2-\delta}$ centered at the seed nodes. Denote this interval as $\mathcal{I}$ and let $\mathcal{N}(\mathcal{I})$ denote the number of missing cycle edges in this interval. Recalling $\eta=n^{\delta}$, we observe that $\mathcal{N}(\mathcal{I}) \sim \operatorname{Bin}(L, 1-\exp (-\eta / 2 n))$ and thus $\mathbb{E}[\mathcal{N}(\mathcal{I})]=L \frac{\eta}{2 n}(1+o(1))=\varepsilon \sqrt{n} / 2(1+o(1))$. Thus we have, by Lemma S4.3

$$
\mathbb{P}\left[\mathcal{N}(\mathcal{I})>\frac{1}{2} \mathbb{E}[\mathcal{N}(\mathcal{I})]\right] \geq 1-2 \exp \left(-\frac{\varepsilon}{24} \sqrt{n}\right)
$$

Note that the waiting time for the simple contagion along the cycle edges to cover the interval $\mathcal{I}$ is distributed as $\sum_{i=1}^{\mathcal{N}(I)} g_{i}$, where $\left\{g_{i}: i \geq 1\right\}$ are iid geometric random variables with mean $1 / q_{n}$. For $\delta>0$ small enough, using (7, Theorem 3.1), we have,

$$
\mathbb{P}\left[\sum_{i=1}^{\mathcal{N}(\mathcal{I})} g_{i}<\delta \frac{\mathcal{N}(\mathcal{I})}{q_{n}} \mid \mathcal{N}(\mathcal{I})\right] \leq \exp \left(-\frac{\delta}{2} \mathcal{N}(\mathcal{I})\right)
$$

Thus with high probability as $n \rightarrow \infty$, for simple contagion along the cycle to cover $\mathcal{I}$, the waiting time to cross the single edges is at least $\varepsilon \delta \sqrt{n} / 4 q_{n}$. Further, Lemma S4.3 implies that the number of missing $\mathcal{C}_{2}$ edges is $O(\sqrt{n} \log n)$ with high probability. As a consequence, for $\delta<1, n^{3 / 2-\delta}-\mathcal{N}(\mathcal{I})=n^{3 / 2-\delta}(1-o(1))$ with high probability. For complex contagion to spread along the cycle, the spreading time required is $\Theta\left(n^{3 / 2-\delta}\right)$ with high probability. Thus in time $t=o\left(\sqrt{n} / q_{n}+n^{3 / 2-\delta}\right)$, with high probability as $n \rightarrow \infty$, contagion along the cycle covers only a subset of the vertices in $\mathcal{I}$.

Finally, we will prove that with high probability as $n \rightarrow \infty$, no vertex outside $\mathcal{I}$ is infected by complex contagion along the long ties. To this end, note that a vertex is infected if it has at least two edges to the infected nodes. The probability of infection of each node is upper bounded by $\mathbb{P}\left[\operatorname{Bin}\left(L, 1-\exp \left(-\eta / n^{2}\right)\right) \geq 2\right]$. Thus the expected number of infected vertices is at most $n \mathbb{P}\left[\operatorname{Bin}\left(L, 1-\exp \left(-\eta / n^{2}\right)\right) \geq 2\right]=O\left(\varepsilon^{2}\right)$. The required claim follows using Markov inequality, once we note that $\varepsilon>0$ is arbitrary. This completes the proof.

The following is an immediate consequence of Theorem S4.4 to lower-bound the spreading time for all values of $1 / 2<\delta<1$. 
Corollary S4.5 (Lower-bounding the spreading time). For any $\varepsilon>0$ and $1 / 2<\delta<1$ with high probability as $n \rightarrow \infty$, up to time $n^{1 / 2-\varepsilon} / q_{n}$, the number of infected nodes is at most $\sqrt{n}$.

The next theorem derives an upper bound to the spreading time for the infection in the regime $\frac{1}{2}<\delta<1$.

Theorem S4.6 (High probability upper-bound on the spreading time). Fix $\delta \in\left(\frac{1}{2}, 1\right)$. Then with high probability as $n \rightarrow \infty$, all nodes are infected by time $3\left(\sqrt{n} / q_{n}+n^{3 / 2-\delta}\right)(\log \log n)^{2}(1+o(1))$.

Proof of Theorem S4.6. The proof is similar to that of Theorem S3.5 and thus we only sketch the proof, focusing on the differences. The proof again proceeds in two stages - in the first stage, we lower bound the growth of infected nodes in the original process and establish that $\Theta\left(n^{5 / 4-\delta / 2} \sqrt{\log n}\right)$ vertices are infected in about $O\left(\left(n^{3 / 2-\delta}+\sqrt{n} / q_{n}\right) \log \log n\right)$ steps. An independent argument establishes that all vertices are infected in $O\left(n^{3 / 2-\delta}+\sqrt{n} / q_{n}\right)$ additional time. Formally, we establish the following results.

Lemma S4.7. For any fixed $\delta \in\left(\frac{1}{2}, 1\right)$ and $C>1$, there exists a sequence $t_{n}:=t_{n}(C, \delta)=o(\log \log n)$ such that with high probability as $n \rightarrow \infty$, the infection spreads to $C n^{5 / 4-\delta / 2} \sqrt{\log n}$ nodes in at most $3\left(n^{3 / 2-\delta}+\right.$ $\left.\sqrt{n} / q_{n}\right) \log \log n\left(\log \log n+t_{n}\right)$ steps.

Lemma S4.8. Let $T_{n}$ be the first time when the contagion infects $C n^{5 / 4-\delta / 2} \sqrt{\log n}$ nodes, where $C>1$ is a universal constant. Then with high probability, all nodes are infected by time $T_{n}+2\left(\frac{\sqrt{n}}{q_{n}}+n^{3 / 2-\delta}\right)$.

The proof follows.

Proof of Lemma S4.7. As before, we divide the $[n]$ nodes into $N$ equal intervals of length $L=n^{3 / 2-\delta}(\log \log n)^{3}$ each. We call an interval active if it contains at least one infected node. At time zero, we call the interval containing the original infected nodes active. We will undercount the original growth of infected vertices by a more tractable growth process. To this end, we look at the infection process at epochs of size $\left(n^{3 / 2-\delta}+\sqrt{n} / q_{n}\right) \log \log n$, and index the epochs by $\tau \geq 1$. During each epoch, the active intervals gain new infected nodes due to infections (simple and complex contagions) along the cycle edges. At the end of an epoch, we expose the long range random edges. This in turn, leads to new active intervals, and provides a lower bound on the growth of infected nodes in the original process. We denote the natural filtration associated with the undercounting process as $\mathcal{F}_{\tau}$, and let $X_{\tau}$ denote the number of active intervals after $\tau$ epochs. In each epoch, with high probability, an infected node further infects its neighbor within $2 \log n / q_{n}$ time. Once we have two neighboring infected vertices, infection spreads along the cycle via simple and complex contagion. Consider the one-sided interval of length $\frac{1}{2} n^{3 / 2-\delta} \log \log n$ on any active interval (to one side if the infected segment on the active interval). Lemma S4.3 implies that with (exponentially) high probability, the maximum number of missing cycle edges is $\frac{1}{2} \sqrt{n} \log \log n$. The total weight time to cross these missing edges is $\sqrt{n} \log \log n / q_{n}$, with (exponentially) high probability. Union bound provides that, with high probability, the contagion process along the cycle infects at least $\frac{1}{2} n^{3 / 2-\delta} \log \log n$ new nodes in each active interval in each epoch of size $\left(n^{3 / 2-\delta}+\sqrt{n} / q_{n}\right) \log \log n$. Note that given $\mathcal{F}_{\tau-1}$,

$$
X_{\tau}-X_{\tau-1}=\operatorname{Bin}\left(N-X_{\tau-1}, p_{\tau}\right) .
$$

where $p_{\tau}$ is the conditional probability of activation of an inactive interval due to the edge-exposure step at this round. We can lower bound this probability as

$$
p_{\tau} \geq \mathbb{P}\left[\operatorname{Bin}\left(L, \mathbb{P}\left[\operatorname{Bin}\left(\frac{X_{\tau-1}}{2} n^{3 / 2-\delta} \log \log n, 1-\exp \left(-\eta / n^{2}\right)\right) \geq 2\right]\right) \geq 1\right] .
$$

Thus conditional on $\mathcal{F}_{\tau-1}$, we have the lower bound

$$
\mathbb{E}\left[X_{\tau}-X_{\tau-1}\right] \geq\left(N-X_{\tau-1}\right) L\left(\frac{X_{\tau-1}}{2} n^{3 / 2-\delta} \log \log n\left(\frac{\eta}{n^{2}}\right)\right)^{2} \geq \frac{1}{8} X_{\tau-1}^{2}(\log \log n)^{2}
$$

Using Bernstein inequality, we can guarantee that this growth is sustained over the first $O(\log \log n)$ epochs leading to $X_{\tau_{n}}>C n^{\delta / 2-1 / 4} \sqrt{\log n}$, for $\tau_{n}=\left(\log \log n+t_{n}\right) / \log 2$ and appropriate choice of $t_{n}=t_{n}(C, \delta)=o(\log \log n)$, exactly as outlined in the proof of Lemma S3.6. The proof now follows using the same arguments as outlined in the proof of Lemma S3.6.

Proof of Lemma S4.8. Fix a node $x$ and let $\mathcal{L}_{x}$ be the interval of length $L=n^{3 / 2-\delta}$ centered at $x$. The probability that any fixed vertex in this interval has at least two infected neighbors is at least $\mathbb{P}\left[\operatorname{Bin}\left(C n^{5 / 4-\delta / 2} \sqrt{\log n}, 1-\exp \left(-\eta / n^{2}\right)\right) \geq\right.$ 2 ]. Using independence of the edges, the probability that no vertex in the interval has at least two infected neighbors 
is at most $\left(1-\mathbb{P}\left[\operatorname{Bin}\left(C n^{5 / 4-\delta / 2} \sqrt{\log n}, 1-\exp \left(-\eta / n^{2}\right)\right)=2\right]\right)^{n^{3 / 2-\delta}}$. Denoting this event as $A_{x}$, simple computations implies that,

$$
\mathbb{P}\left(A_{x}\right) \leq n^{-C}
$$

for $C>1$. By a union bound, with high probability, each node has at least one infected node in the interval of length $L$ surrounding it. With exponentially high probability, an infected node further infects its neighbor within $2 \log n / q_{n}$ time. Using union bound (over $n$ choices of $x$ ), in $2(\log n) / q_{n}$ additional time, every interval $\mathcal{L}_{x}$ will have two neighboring infected vertices and infection spreads throughout the $\mathcal{L}_{x}$ intervals via simple and complex contagion. Next note that using Lemma $\mathrm{S} 4.3$ and a union bound over $x$, the maximum number of missing $\mathcal{C}_{2} \backslash \mathcal{C}_{1}$ edges in any interval $\mathcal{L}_{x}$ is at most $\sqrt{n}$. Further, an application of $(6$, Theorem 1.14) and union bound (over $x$ ) implies that the total passage time across the missing edges in any interval $\mathcal{L}_{x}$ is at most $2 \sqrt{n} / q_{n}$. Finally, complex contagion covers the remaining part of each interval in less than $n^{3 / 2-\delta}$ steps. Thus with high probability, the entire graph is infected by time $T_{n}+2\left(\sqrt{n} / q_{n}+n^{3 / 2-\delta}\right)$. 
C. Simulation results allowing for simple adoptions along all edges. Figure S2 is a redone of the simulation results presented in Figure 4C of the main text, allowing for simple contagion probability $q$ along all edges in the network. Both figures reveal the same kind of qualitative behavior in that, rewiring accelerates the spread for large enough values of $q$. Moreover, comparisons of the two figures reveal interesting consequences of the handicap assumption that we impose on the model in Figure $4 \mathrm{C}$, by allowing simple contagions only along the $\mathcal{C}_{1}$ edges.

Not only the spread is accelerated for much smaller values of simple adoption probability $(q \geq 0.0129)$ in Figure S2 compared to Figure 4C, but also the initial slow down phase is no longer present in Figure S2. To the contrary, the first few rewired edges are the most effective in accelerating the spread: we observe diminishing returns in the acceleration (or deceleration) of contagion with the number of rewired edges in Figure S2.

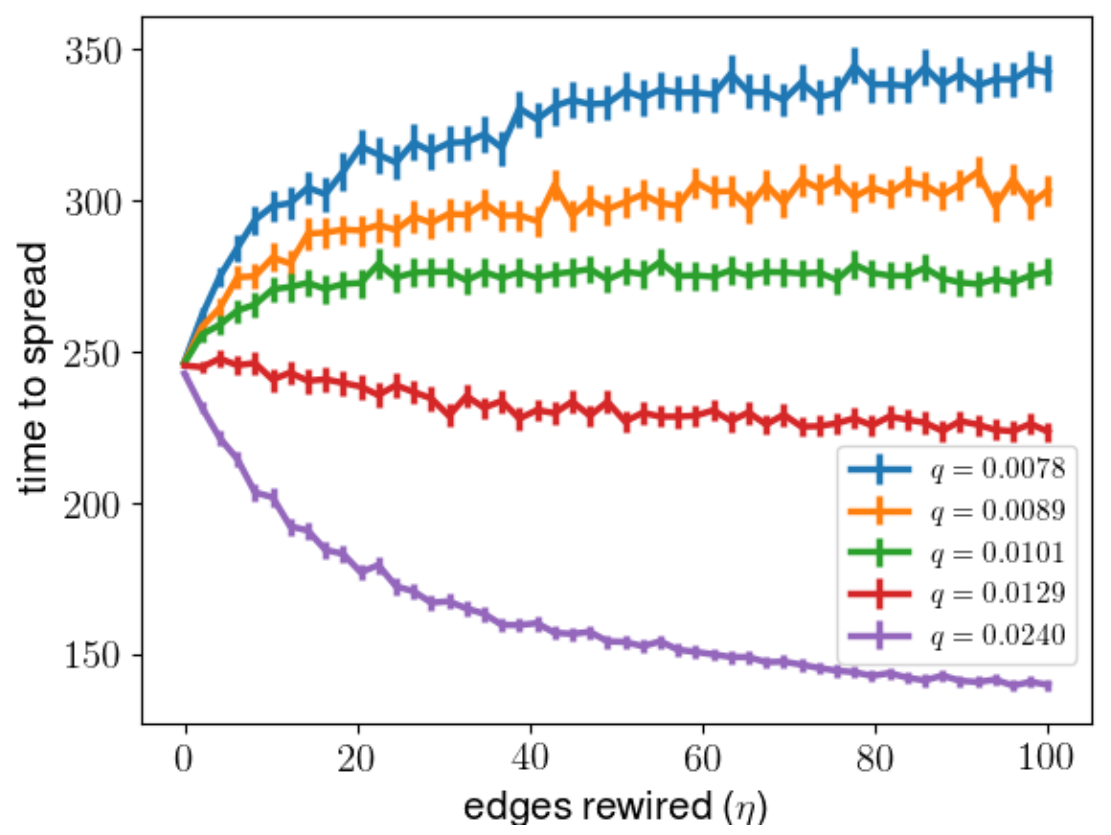

Fig. S2. Spreading time of complex contagion over $\mathcal{C}_{2}^{\eta}$ random graphs with $n=500$, allowing for simple contagion with probability $q$ along all edges (not just $\mathcal{C}_{1}$ ). Each point is the average of 1000 random draws and the error bars show the $95 \%$ normal confidence intervals around the means. 


\section{S5. Simulations with empirical networks data}

We expand on the results of simulations that we present in the main text (Figure 6) by providing additional statistics and measurements over these networks. Moreover, we provide simulations with variations of the original model to verify the robustness of our claims to changes in the model. We use four datasets that contain empirical social networks for (i) 175 rural Chinese farm villages being encouraged to sign up for insurance (8), (ii) the union of the friendship and the health advice networks collected from 17 rural villages in Uganda (9), (iii) 77 village social networks collected in the study of participation in a microfinance program in South India (10), and (iv) the Facebook friendship social networks in U.S. universities and colleges (11), from which we choose the 40 smallest networks. Table S1 summarizes the network size statistics in all these datasets. For each social network in these datasets we are interested in how interventions that modify the network structure would affect the spread of contagion. We consider three intervention strategies: (i) random rewiring, (ii) adding new edges uniformly at random, (iii) adding new edges at random with probability proportional to the number of open triads that each new edge would close. Following the same conventions as in Figure 6 of the main text, we use black, orange, blue, and red colors to plot the values corresponding to the original network, as well as the modified networks under rewiring, triad-closing and random edge additions. The plots in Figure S3 are produced in the same way as Figure 6B of the main text. The latter is simulated over the 175 social networks in the Cai et al. (8) dataset, whereas in Figure S3 we use the other three datasets with fewer social networks - Chami et al. (9), Banerjee et al. (10), and Traud et al. (11).

Table S1. Size statistics for the empirical networks data

\begin{tabular}{lrrrrr} 
dataset & number of networks & min size & max size & median size & mean size \\
\hline Cai et al. (8) & 175 & 13 & 117 & 51.5 & 54.03 \\
Chami et al. (9) & 17 & 65 & 372 & 184 & 202.12 \\
Banerjee et al. (10) & 77 & 75 & 341 & 189 & 192.63 \\
Traud et al.(11) & 40 & 762 & 7677 & 3745 & 4153.82 \\
\hline
\end{tabular}

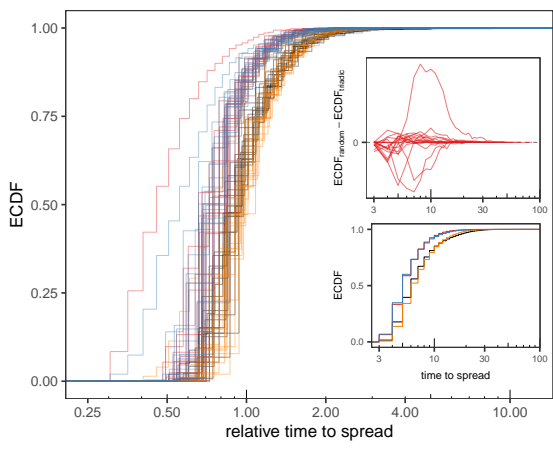

(A) Uganda Rural Villages (9)

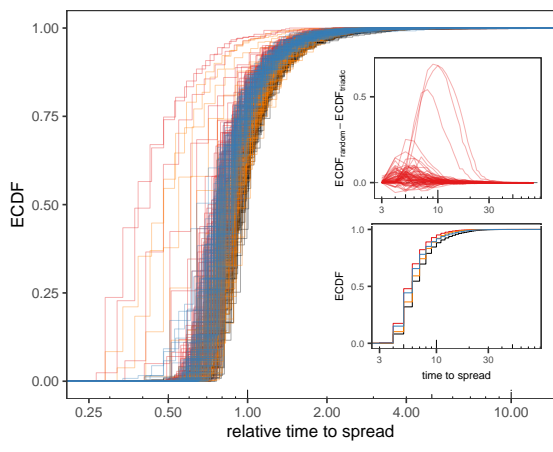

(B) South Indian Villages (10)

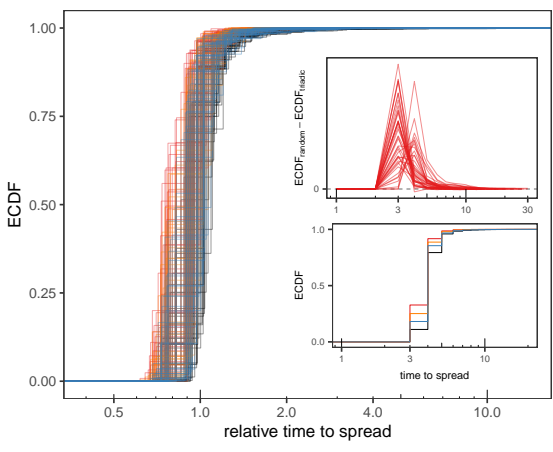

(C) Facebook Friendship Networks (11)

Fig. S3. We replicate the plots in Figure 6B of the main text (that was simulated on social network data from Chinese farm villages (8)) for three other datasets.

In any given network, starting from two randomly chosen seeds (initially infected nodes) we measure the time until $90 \%$ of the nodes are infected. Under each intervention and model of contagion that we are considering, we draw 500 random spreading time samples over the original or modified networks and compare the spreading times in terms of their empirical cumulative distribution functions (ECDFs). On a few occasions we combine these samples for all the villages over the entire dataset and compare the overall ECDFs under each intervention: Figures S5, S6, as well as the bottom left insets in Figure S3, and the second and forth rows in Figure S7 are generated this way. The intervention sizes are measured in terms of the percent of edges in the original networks, and unless otherwise specified, the intervention size is fixed at 10\%: Figure 6 in the main text, as well as Figures S5 and S8, here, follow this convention. When varying the intervention size, we group the samples by their intervention size. The intervention size in each group is fixed at 5, 10, 15, 20 or 25\%: Figures S6 and S7 are generated in this manner. In the first and third rows of Figure S7 we have overlaid the ECDFs of spreading times for each social network in the respective datasets (77 villages from the south Indian dataset (10) in the first row, and 17 villages from the Ugandan dataset (9) in the third row). In these two cases (the first and third rows of Figure S7) since different village sizes affect the time 

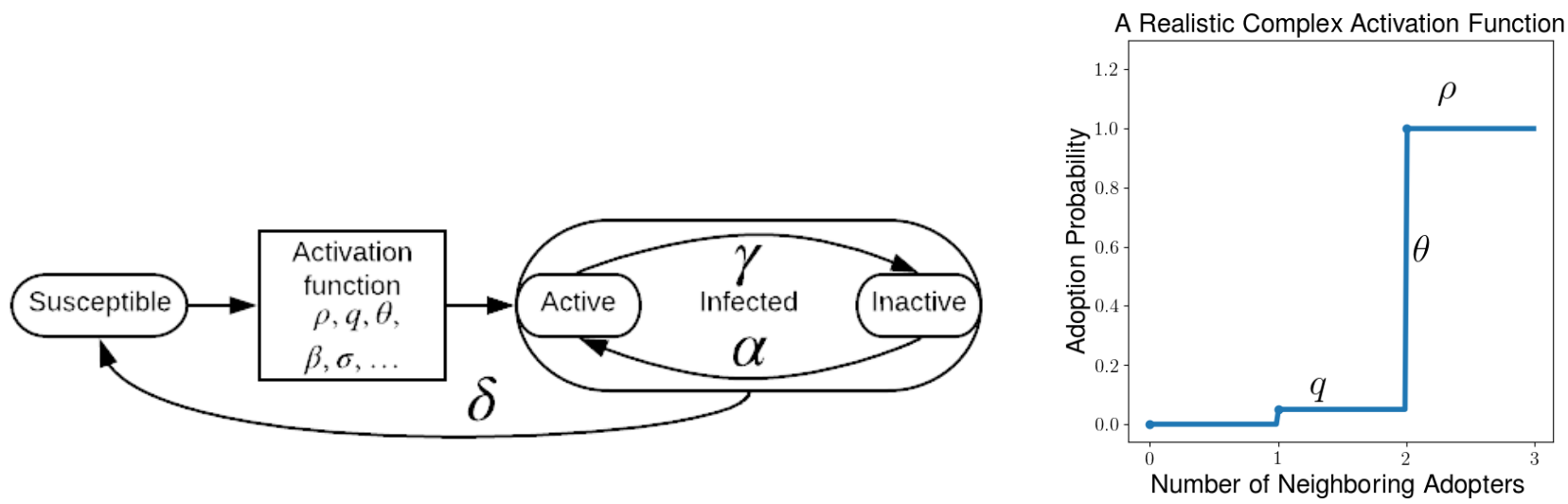

Fig. S4. The model

to $90 \%$ spread, we normalize the spreading times relative to the mean spread time in the original village (with no interventions); moreover, we put the x-axis in logarithmic scale to symmetrize the ratios below and above one.

Figure S4 shows the block diagram of the model that we have implemented for each agent in our simulations. The active and inactive infected states describe a situation where adopter agents may transition into a state where they are not effective in turning their neighboring non-adopters into adopters. We refer to this state as an inactive infected state and allow infected agents to transition back and forth between active and inactive states with probabilities $\alpha$ and $\gamma$ as depicted in Figure S4. In the basic model that we study in the main text all infected agents are regarded as active; hence, $\alpha=1$ and $\gamma=0$. In general, we also allow a probability $\delta$ for infected agents to transition back into the susceptible state; however, in our studies we set $\delta=0$, as motivated by applications where infection connotes product purchase.

The activation function block determines the type of contagion (simple or complex). Different models of contagion are characterized by different parameters. For example, the simple contagion activation functions shown in Figure 1 of the main text are parametrized by the independent transmission probabilities $(\beta)$ along each edge. The probability of infection with $x$ infected neighbors under this simple contagion activation function is given by $1-(1-\beta)^{x}$. On the other hand, the complex contagion activation function shown in Figure S4, on the right, is parametrized by a threshold value $\theta$, adoption probability above threshold $\rho$, and adoption probability below threshold $q$. Similarly, logit and probit activation functions studied in Section S3C are characterized by a threshold $\theta$ and noise (or "rationality") level $\sigma$. Unless otherwise specified, we compute the spreading time samples under the $(0.05,1)$ model from Figure S5A $(\rho=1, q=0.05, \alpha=1, \gamma=0, \theta=2)$ : the spreading time samples in Figures S7 and S8, here, as well as Figure 6 of the main text are all generated under the $(0.05,1)$ model.

In special cases (Figure S5D, here, and Figure 4C in the main text) we make a distinction between what edges pass simple contagion adoptions (probability $q$ ) and what edges do not. Such models are not fully specified by the choice of activation functions and transition probabilities $\alpha, \gamma$, and $\delta$, since we need to take into account the type of network edges along which transitions occur. In Figure S5D, we allow the simple contagion probability $q$ only if the edge connecting the adopter and non-adopter agent is existent in the original network. Spreading time samples for this model are collected only under the edge addition interventions (random versus triad-closing). In Figure 4C of the main text we consider the spreading time over $\mathcal{C}_{2}^{\eta}$ random graphs and allow for simple contagion probability $q$ only along the $\mathcal{C}_{1}$ cycle edges.

Figure S5 shows the overall ECDFs (combining all samples across all villages with 500 random samples per village) of the spreading times over the Chinese farm villages dataset (8). We present the results for six different models and activation function settings with $10 \%$ sized interventions. A more fine-grained version of this data where intervention sizes are varied between $5,10,15,20$, and $25 \%$ is presented in Figure S6. The two models labeled by $(0.05,1(0.05,0.5))$ and $(0.001,1)$ in Figures S5C and S5F (see the Figure captions for the corresponding model parameters) are significantly slower. In their ECDFs, we can identify a fast and a slow regime which we attribute to complex and simple contagions, respectively. If the $90 \%$ spread is reliant on complex contagion, then the spreading time samples are visibly discrete and the total spread is achieved fast. If, on the other hand, a significant number of nodes are infected through simple contagion (coin flips with probability $q$ ), then contagion is slow and spreading time samples cover a wide range, resulting in a smooth section in the ECDF curve.

In contrast, the fractional threshold model that is labeled by $\operatorname{REL}(0.05,1)$ in Figure S5E is significantly faster; its 
ECDF is visibly discrete and does not contain a smooth, slow section. Under this fractional model a node is infected with high probability $(\rho=1)$ if the majority of its neighbors are adopters $\left(\theta^{\star}=0.5\right)$; in particular, a leaf node with only one neighbor becomes an adopter as soon as its neighbor adopts. This is in contrast to the absolute threshold model $\theta=2$ where a leaf node can only be infected through the slow simple contagion (coin flips) with probability $q$.

In Figure S7 we present the ECDFs of the spreading times over the south Indian (10) and Ugandan (9) villages for different intervention sizes (the intervention size in Figure S3 was fixed at 10\%). To further demonstrate the stochastic dominance relation between the spreading times (as random variables) under various interventions, in Figure S8 we present the difference between the ECDFs with $10 \%$ interventions for all the social networks in our four datasets. The spreading samples in both Figures S7 and S8 are generated under the $(0.05,1)$ model from Figure S5A $(\rho=1, q=0.05, \alpha=1, \gamma=0, \theta=2)$. If the positive difference persists throughout the entire support of two ECDFs, then we conclude the stochastic dominance relation between their respective random variables.

In Figure S9 we plot the mean time to spread versus the intervention size for random and triadic edge additions. We can thus answer how much larger does the intervention size for triadic addition have to be before it is faster than random addition in each set of empirical networks. For example, Figure S9A indicates that in the Cai et al. dataset even with $25 \%$ additional triad-closing ties the mean spread time is still slower than in the networks with $10 \%$ additional random ties.

In all these measurements, we observe the same direction (sign) for the effect of interventions on the spreading time: (i) rewiring speeds up the spread of contagion compared to the original networks, (ii) contagion spreads faster in the networks with added edges compared to the original networks, (iii) contagion spreads faster in the network with added random edges compared to the network with added triad-closing edges. The magnitude of differences are larger for larger interventions. 


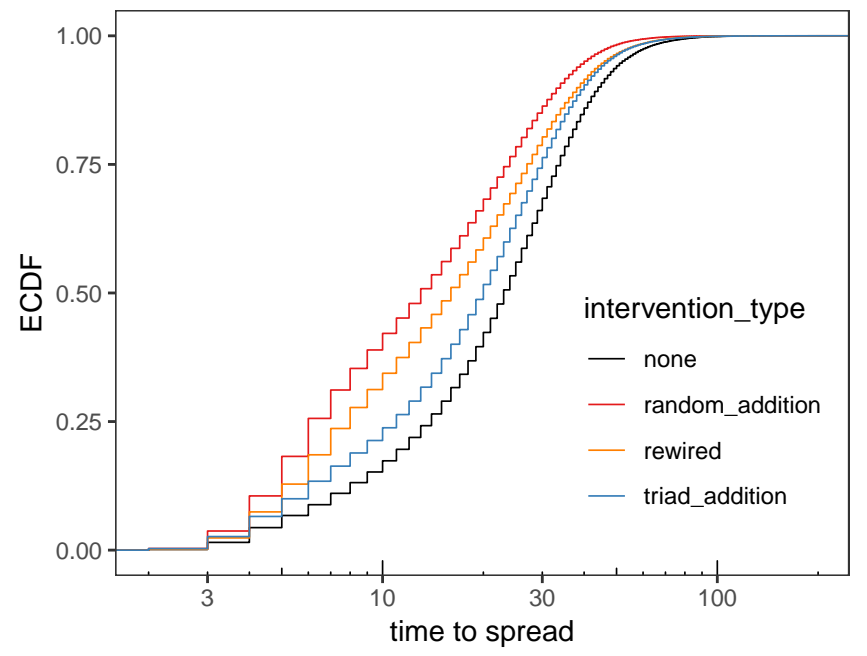

(A) $(0.05,1): \rho=1, q=0.05, \alpha=1, \gamma=0, \theta=2$

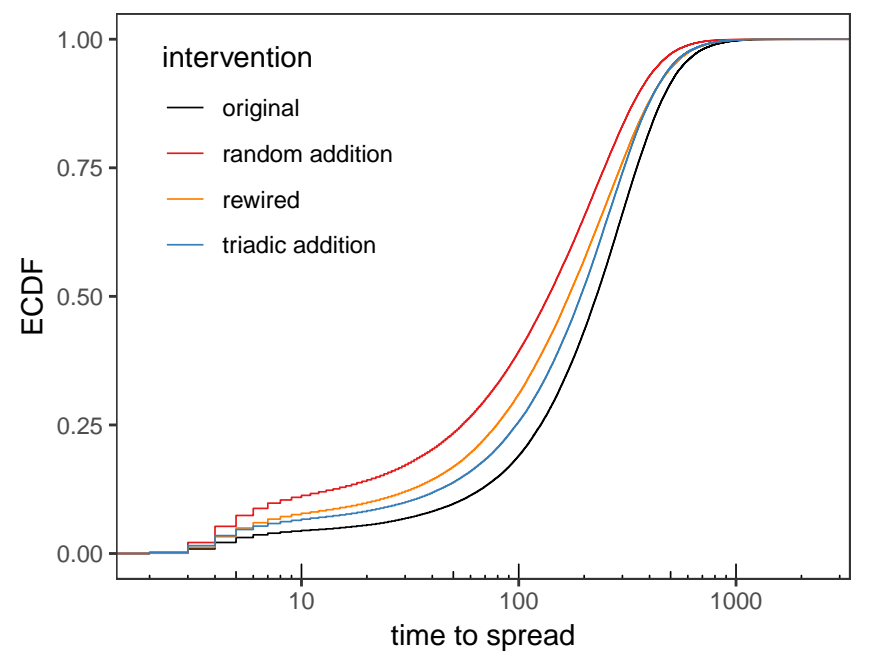

(C) $(0.05,1(0.05,0.5)): \rho=1, q=0.05, \alpha=0.05, \gamma=0.5, \theta=2$

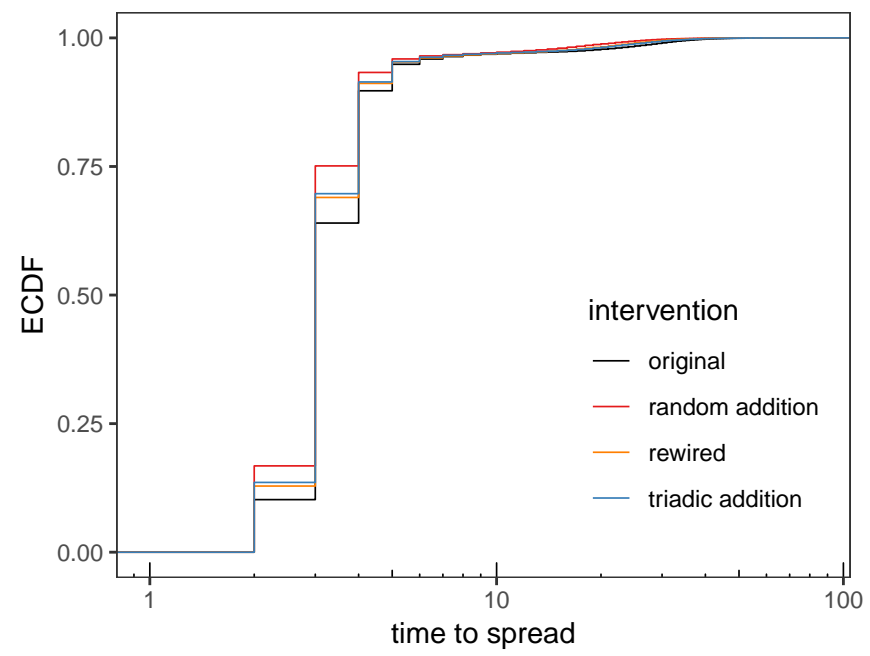

(E) REL $(0.05,1): \rho=1, q=0.05, \alpha=1, \gamma=0, \theta^{\star}=0.5$, complex contagion with fixed common fractional threshold $\left(\theta^{\star}\right)$

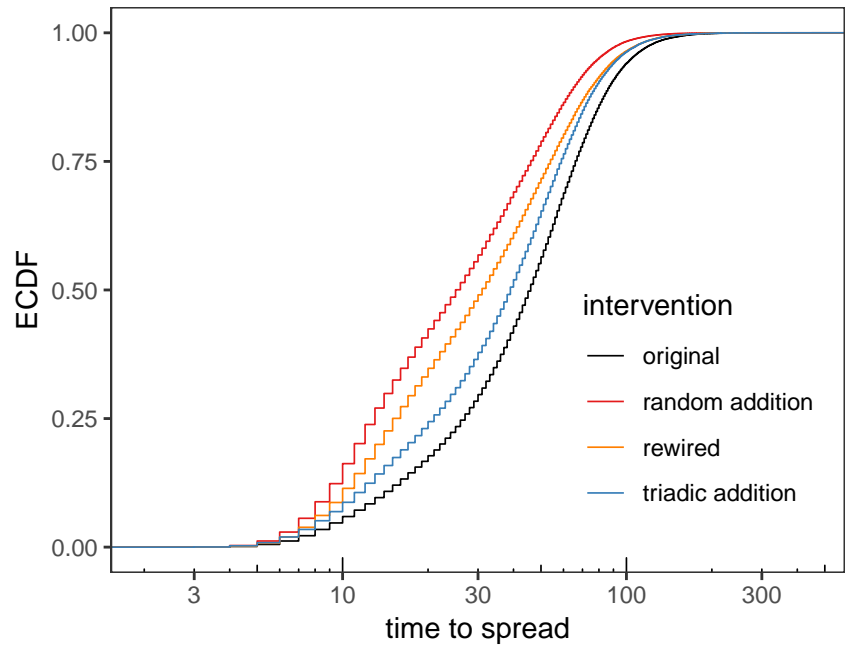

(B) $(0.025,0.5): \rho=0.5, q=0.025, \alpha=1, \gamma=0, \theta=2$

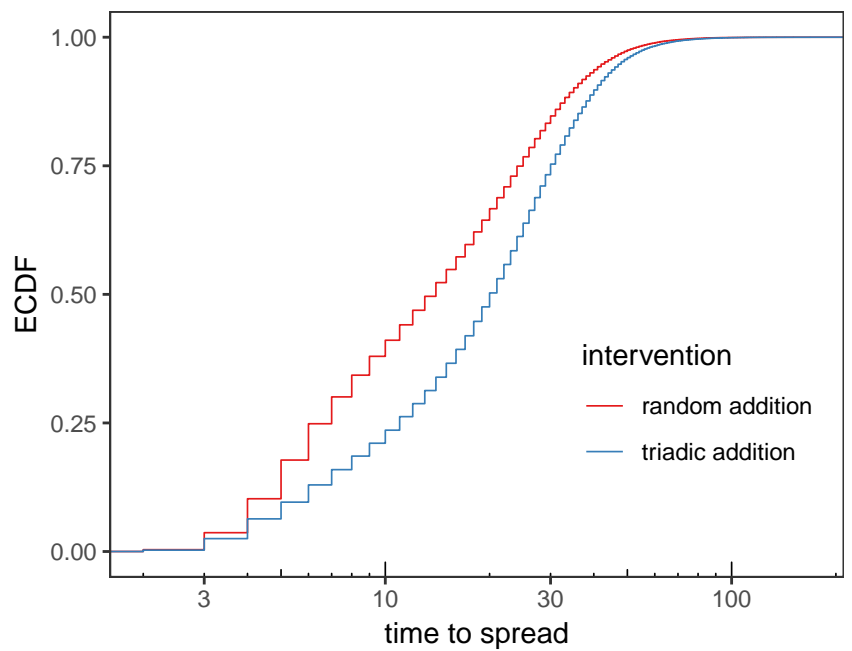

(D) (ORG-0.05,1): $\rho=1, q=0.05, \alpha=1, \gamma=0, \theta=2$, simple adoptions happen only along the original edges and spreading time samples are collected only under edge addition interventions (triadic vs random)

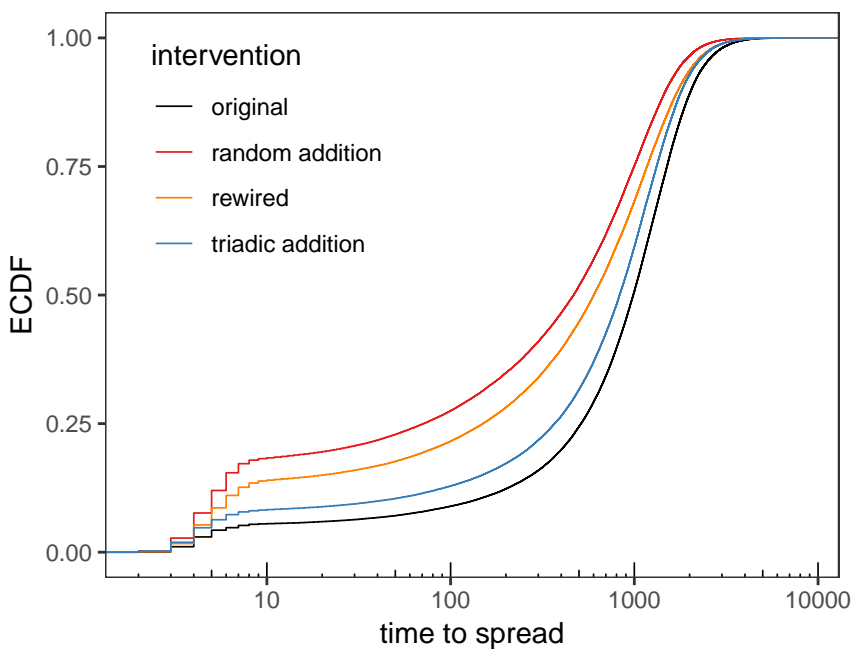

(F) $(0.001,1): \rho=1, q=0.001, \alpha=1, \gamma=0, \theta=2$

Fig. S5. The overall ECDF for the spreading times over Chinese farm villages (8) under different contagion models and interventions with fixed size (10\%) 


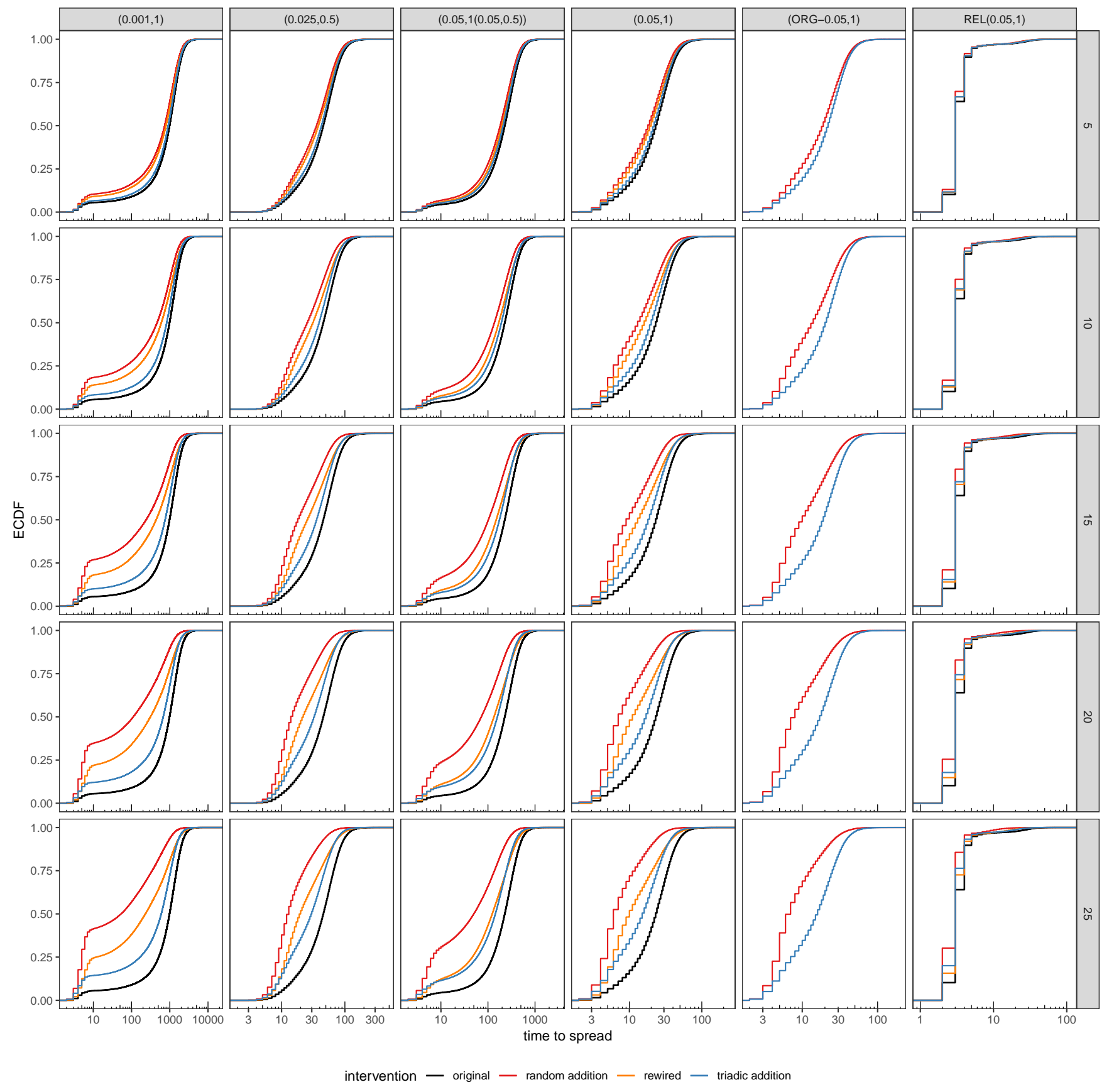

Fig. S6. Overall ECDFs under various interventions grouped by the intervention sizes and contagion models for the Chinese farming villages (8). The model labels at the top are as introduced in the captions of Fig. S5. The intervention sizes are shown on right side. 

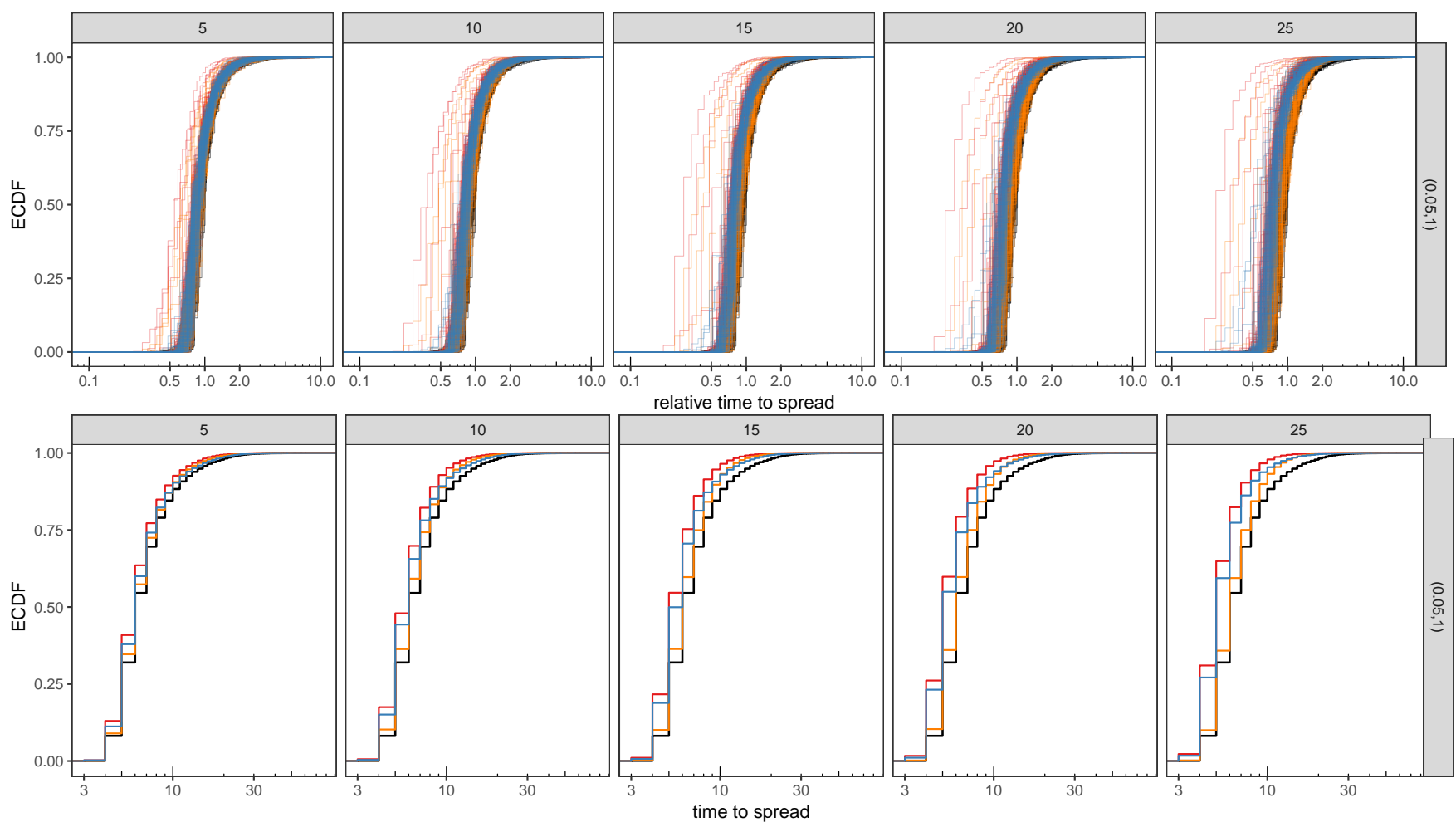

intervention_type - none $—$ random_addition — rewired — triad_addition
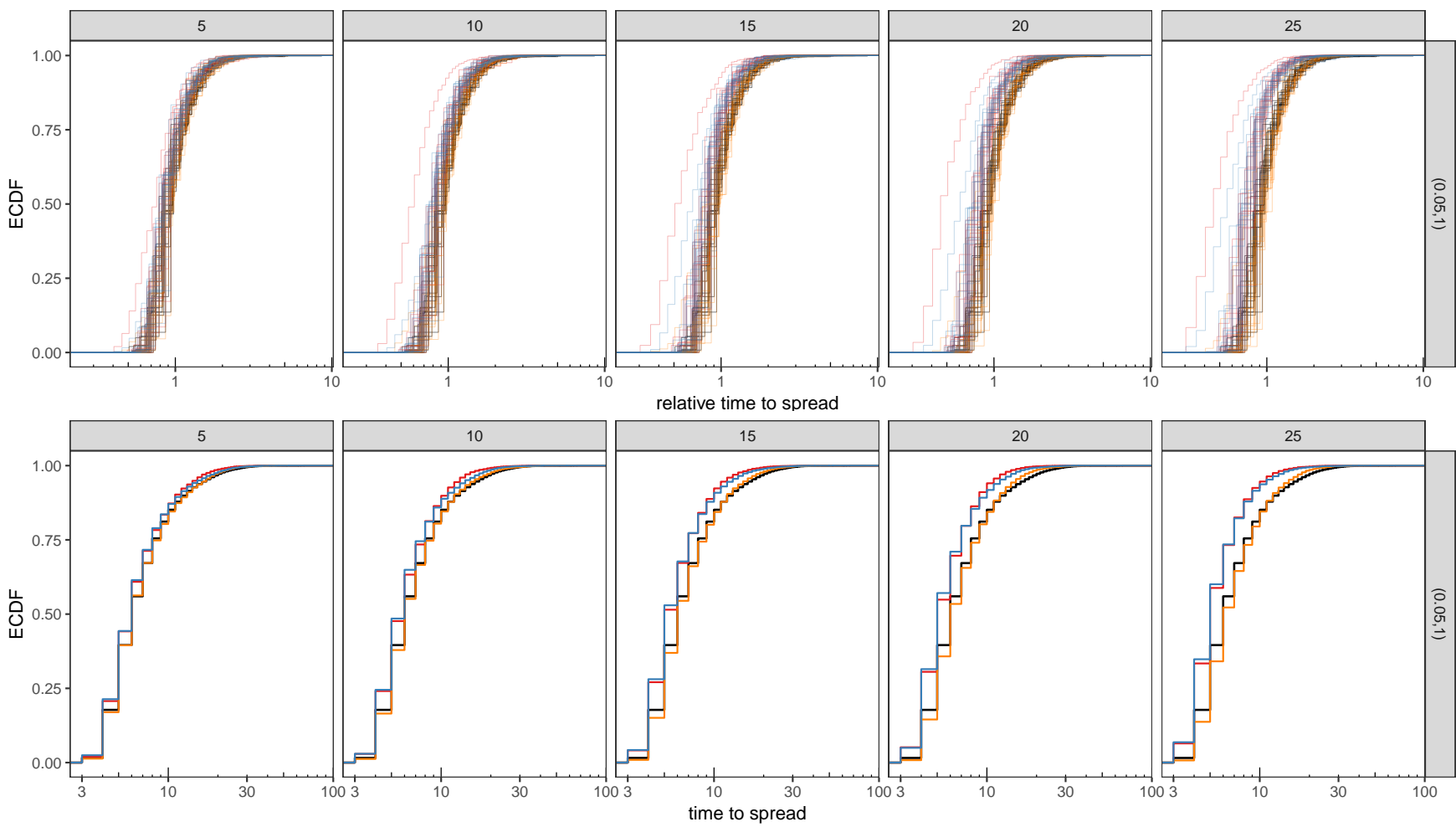

intervention_type — none — random_addition — rewired — triad_addition

Fig. S7. ECDFs under various interventions grouped by the intervention size for the South Indian (10, top two) and Ugandan (9, bottom two) villages. For each dataset, the top figures show the ECDFs for the individual villages (overlaid) and the bottom figures combine the random samples (spreading times) over the entire dataset. These figures follow the same conventions as Figure 6 of the main text. 

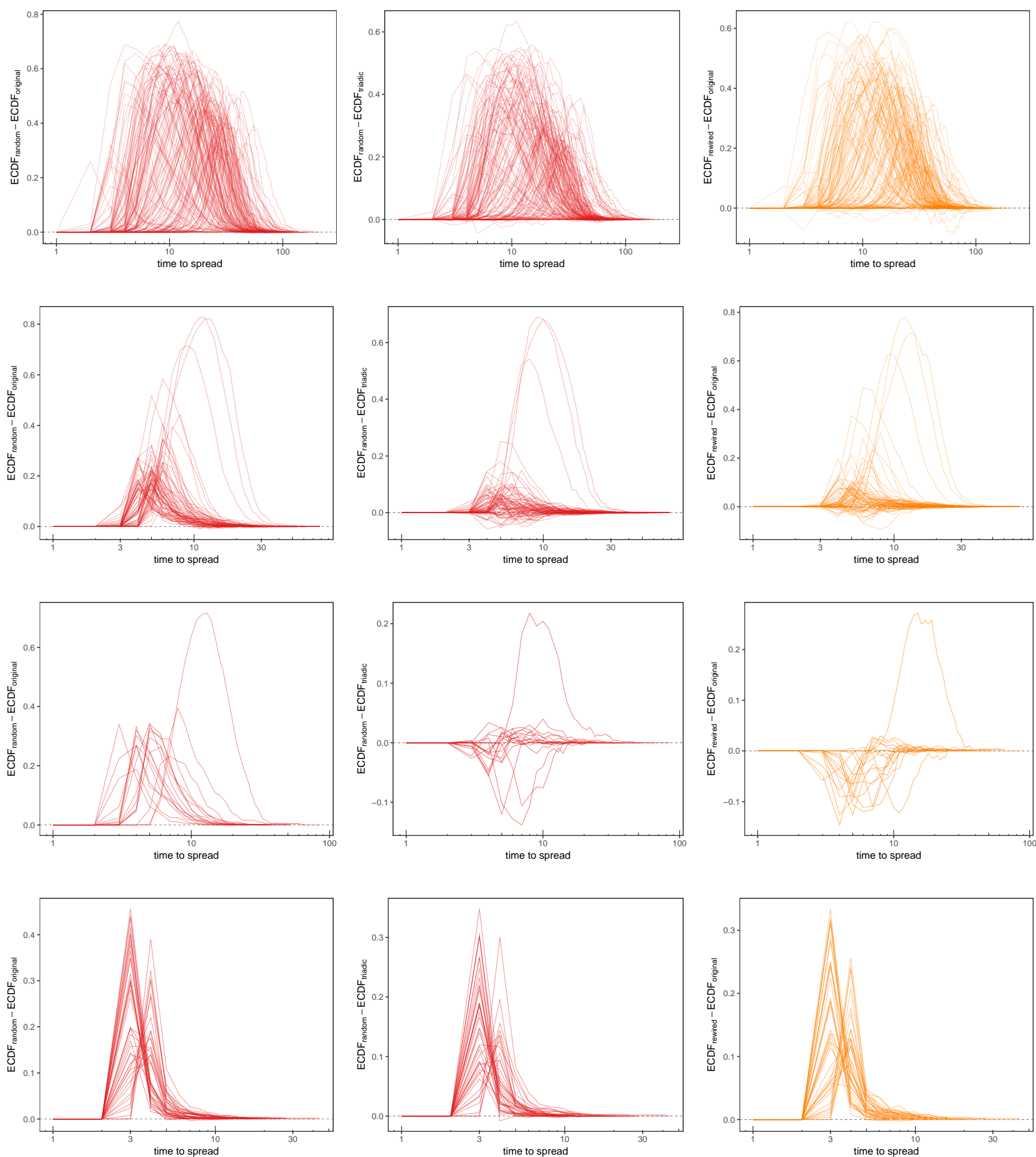

Fig. S8. Difference between spreading time ECDFs in networks with $10 \%$ additional random edges and the original ones (leftmost), between the networks with $10 \%$ additional random and triad closing edges (middle), and between the $10 \%$ rewired and the original networks (rightmost), for rural villages in China (8) - first row, South Indian (10) — second row, and Uganda (9) - third row, as well as the Facebook social networks (11) - fourth row. The positive differences indicate the stochastic dominance relation between the spread times (as random variables) under one intervention over another. The spreading time samples are computed undet the ( $0.05,1)$ model from Figure S5A with $q=0.05, \alpha=1, \gamma=0$, and $\theta=2$. 


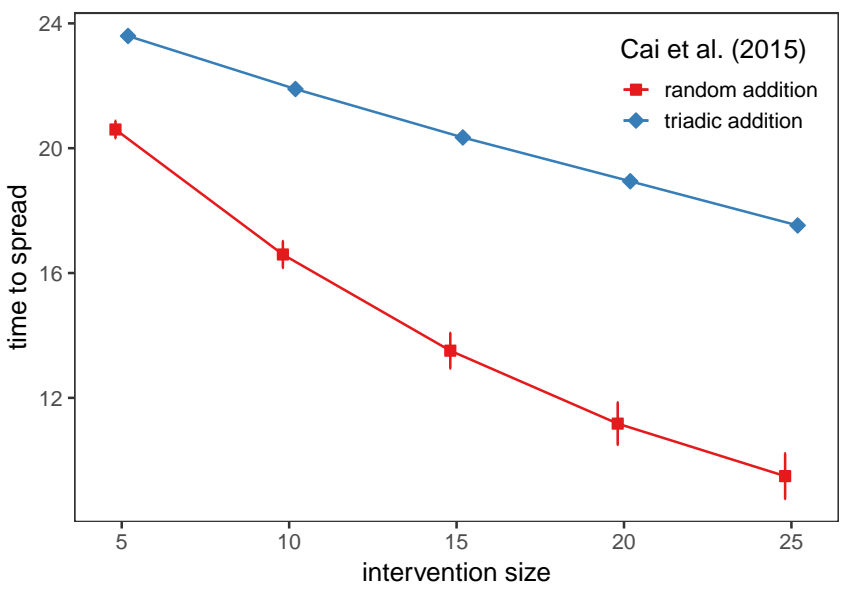

(A)

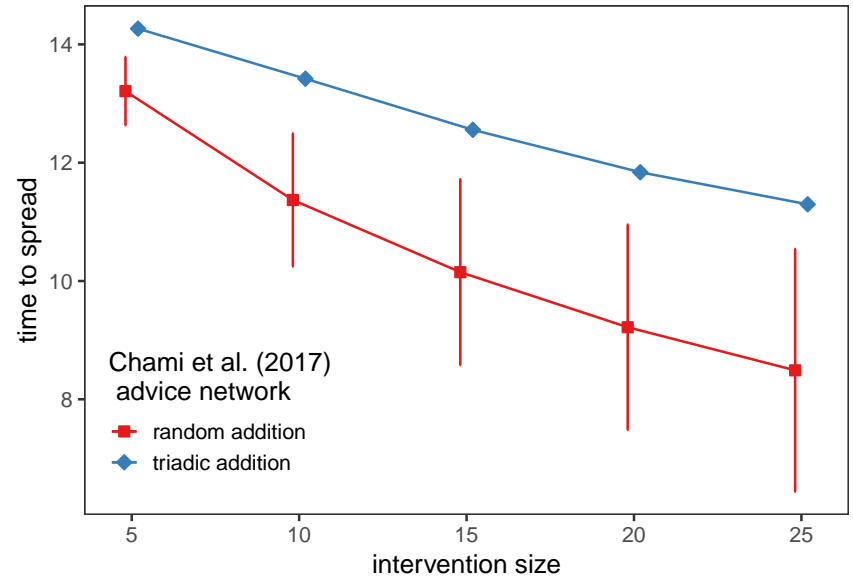

(C)

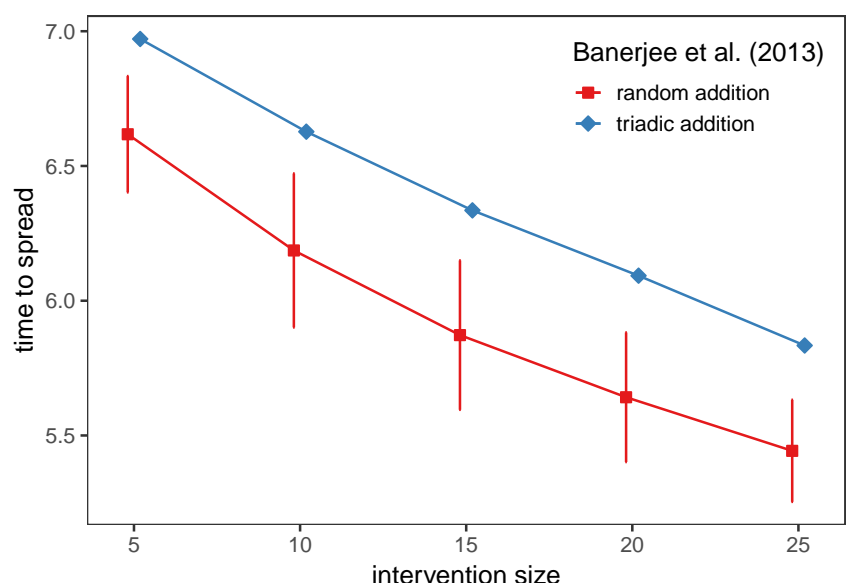

(B)

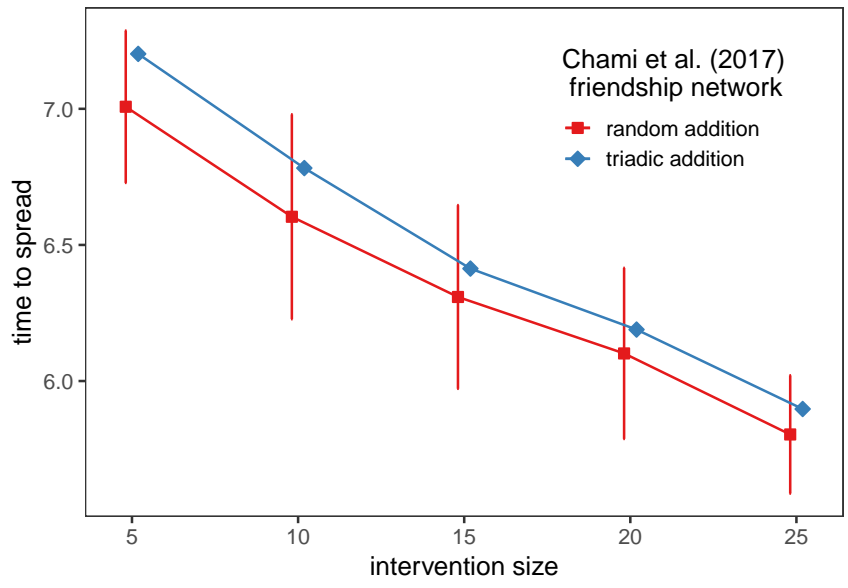

(D)

Fig. S9. We plot the mean time to spread in four sets of empirical networks with added triad-closing or random ties. The x-axis indicates the number of added ties (intervention size) as percent of edges in the original networks. Each point averages over all networks in that set. Error bars are $95 \%$ confidence intervals for the difference from the spread times over the networks with added triad-closing ties. We compute the confidence intervals by treating each network as a single observation. 


\section{References}

1. Aral S, Muchnik L, Sundararajan A (2009) Distinguishing influence-based contagion from homophily-driven diffusion in dynamic networks. Proceedings of the National Academy of Sciences 106(51):21544-21549.

2. Bakshy E, Rosenn I, Marlow C, Adamic L (2012) The role of social networks in information diffusion in Proceedings of the 21st international conference on World Wide Web. (ACM), pp. 519-528.

3. Centola D (2010) The spread of behavior in an online social network experiment. science 329(5996):1194-1197.

4. Mønsted B, Sapieżyński P, Ferrara E, Lehmann S (2017) Evidence of complex contagion of information in social media: An experiment using twitter bots. PloS one 12(9):e0184148.

5. Ugander J, Backstrom L, Marlow C, Kleinberg J (2012) Structural diversity in social contagion. Proceedings of the National Academy of Sciences 109(16):5962-5966.

6. Doerr B (2011) Analyzing randomized search heuristics: Tools from probability theory in Theory of Randomized Search Heuristics: Foundations and Recent Developments. (World Scientific), pp. 1-20.

7. Janson S (2018) Tail bounds for sums of geometric and exponential variables. Statistics 8 Probability letters 135:1-6.

8. Cai J, De Janvry A, Sadoulet E (2015) Social networks and the decision to insure. American Economic Journal: Applied Economics 7(2):81-108.

9. Chami GF, Ahnert SE, Kabatereine NB, Tukahebwa EM (2017) Social network fragmentation and community health. Proceedings of the National Academy of Sciences 114(36):E7425-E7431.

10. Banerjee A, Chandrasekhar AG, Duflo E, Jackson MO (2013) The diffusion of microfinance. Science 341(6144):1236498.

11. Traud AL, Mucha PJ, Porter MA (2012) Social structure of facebook networks. Physica A: Statistical Mechanics and its Applications 391(16):4165-4180. 